\title{
Pulsating semi-waves in periodic media and spreading speed determined by a free boundary model ${ }^{\text {is }}$
}

\author{
Yihong $\mathrm{Du}^{\mathrm{a}, *}$, Xing Liang ${ }^{\mathrm{b}}$

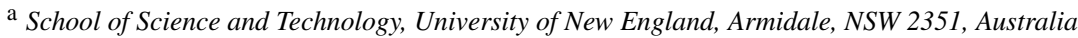 \\ ${ }^{\mathrm{b}}$ Wu Wen-Tsun Key Laboratory of Mathematics and School of Mathematical Science, University of Science and Technology of China, Hefei, \\ Anhui 230026, PR China
}

Received 5 January 2013; received in revised form 1 October 2013; accepted 25 November 2013

Available online 16 December 2013

\begin{abstract}
We consider a radially symmetric free boundary problem with logistic nonlinear term. The spatial environment is assumed to be asymptotically periodic at infinity in the radial direction. For such a free boundary problem, it is known from [7] that a spreading-vanishing dichotomy holds. However, when spreading occurs, only upper and lower bounds are obtained in [7] for the asymptotic spreading speed. In this paper, we investigate one-dimensional pulsating semi-waves in spatially periodic media. We prove existence, uniqueness of such pulsating semi-waves, and show that the asymptotic spreading speed of the free boundary problem coincides with the speed of the corresponding pulsating semi-wave.

(c) 2013 L'Association Publications de l'Institut Henri Poincaré. Published by Elsevier B.V. All rights reserved.
\end{abstract}

MSC: 35K20; 35R35; 35J60; 92B05

Keywords: Diffusive logistic equation; Free boundary; Periodic environment; Pulsating semi-wave; Spreading speed

\section{Introduction}

We are interested in the evolution of the positive solution $u(t, r)\left(r=|x|, x \in \mathbb{R}^{N}, N \geqslant 1\right)$, governed by the following diffusive logistic equation with a free boundary:

$$
\begin{cases}u_{t}-d \Delta u=u(\alpha(r)-\beta(r) u), & t>0,0<r<h(t), \\ u_{r}(t, 0)=0, \quad u(t, h(t))=0, & t>0, \\ h^{\prime}(t)=-\mu u_{r}(t, h(t)), & t>0, \\ h(0)=h_{0}, \quad u(0, r)=u_{0}(r), & 0 \leqslant r \leqslant h_{0},\end{cases}
$$

\footnotetext{
We thank the referee for a very careful reading of the paper and useful suggestions. This work was supported by the Australian Research Council and NSFC 11171319. Part of this research was carried out during a visit of X. Liang to the Univ. of New England in Feb.-March 2011 and a visit of Y. Du to the Univ. of Sci. and Tech. of China in Sept.-Oct. 2012.

* Corresponding author.

E-mail addresses: ydu@turing.une.edu.au (Y. Du), xliang@ustc.edu.cn (X. Liang).
} 
where $\Delta u=u_{r r}+\frac{N-1}{r} u_{r} ; r=h(t)$ is the free boundary to be determined; $h_{0}, \mu$ and $d$ are given positive constants; $u_{0} \in C^{2}\left(\left[0, h_{0}\right]\right)$ is positive in $\left[0, h_{0}\right)$ and $u_{0}^{\prime}(0)=u_{0}\left(h_{0}\right)=0$; the functions $\alpha(r)$ and $\beta(r)$ are positive and satisfy the following conditions:

$$
\left\{\begin{array}{l}
\text { (i) } \quad \alpha, \beta \in C^{\nu_{0}}([0, \infty)) \text { for some } \nu_{0} \in(0,1), \\
\text { (ii) } \text { there exist positive } L \text {-periodic functions } a \text { and } b \text { in } C^{\nu_{0}}(\mathbb{R}) \text { such that } \\
\\
\lim _{r \rightarrow+\infty}(|\alpha(r)-a(r)|+|\beta(r)-b(r)|)=0 .
\end{array}\right.
$$

Problem (1.1) may be viewed as describing the spreading of a new or invasive species with population density $u(t,|x|)$ over an $N$-dimensional habitat, which is radially symmetric, heterogeneous and asymptotically spaceperiodic near infinity in the radial direction. The initial function $u_{0}(|x|)$ stands for the population in its early stage of introduction. Its spreading front is represented by the free boundary $|x|=h(t)$, which is a sphere $\partial B_{h(t)}$ with radius $h(t)$ growing at a speed proportional to the gradient of the population density at the front: $h^{\prime}(t)=-\mu u_{r}(t, h(t))$. (A deduction of this condition based on ecological considerations can be found in [6].) The coefficient functions $\alpha(|x|)$ and $\beta(|x|)$ represent the intrinsic growth rate of the species and its intra-specific competition respectively, and $d$ is the random diffusion rate.

Problem (1.1) was studied recently in [7], and when $\alpha, \beta$ are positive constants and the space dimension is one, this problem was considered earlier in [10]. In both cases, it was shown that a unique solution pair $(u, h)$ exists, with $u(t, r)>0$ and $h^{\prime}(t)>0$ for $t>0$ and $0 \leqslant r<h(t)$, and a spreading-vanishing dichotomy holds, namely, a spatial barrier $r=R^{*}$ exists, such that either

- Spreading: the free boundary breaks the barrier at some finite time (i.e., $h\left(t_{0}\right) \geqslant R^{*}$ for some $t_{0} \geqslant 0$ ), and then the free boundary goes to infinity as $t \rightarrow \infty$ (i.e., $\lim _{t \rightarrow \infty} h(t)=\infty$ ), and the population spreads to the entire space and stabilizes at its positive steady-state, or

- Vanishing: the free boundary never breaks the barrier $\left(h(t)<R^{*}\right.$ for all $\left.t>0\right)$, and the population vanishes $\left(\lim _{t \rightarrow \infty} u(t, r)=0\right)$.

Moreover, when spreading occurs, it follows from Theorem 3.6 of [7] that

$$
\liminf _{t \rightarrow \infty} \frac{h(t)}{t} \geqslant k_{*}, \quad \limsup _{t \rightarrow \infty} \frac{h(t)}{t} \leqslant k^{*}
$$

for some positive constants $k_{*}$ and $k^{*}$ determined by the pairs $\left(\alpha_{\infty}, \beta^{\infty}\right)$ and $\left(\alpha^{\infty}, \beta_{\infty}\right)$, respectively, where

$$
\begin{array}{rlrl}
\alpha_{\infty} & :=\liminf _{r \rightarrow \infty} \alpha(r), & \beta^{\infty}:=\limsup _{r \rightarrow \infty} \beta(r), \\
\alpha^{\infty}:=\limsup _{r \rightarrow \infty} \alpha(r), & \beta_{\infty}:=\liminf _{r \rightarrow \infty} \beta(r) .
\end{array}
$$

It follows that if both $\lim _{r \rightarrow \infty} \alpha(r)$ and $\lim _{r \rightarrow \infty} \beta(r)$ exist, then $\lim _{t \rightarrow \infty} \frac{h(t)}{t}=k$ exists, and one may regard $k$ as the asymptotic spreading speed.

The main purpose of this paper is to show that under condition (1.2), $\lim _{t \rightarrow \infty} \frac{h(t)}{t}$ also exists, and we will use pulsating semi-waves (to be defined below) induced by (1.1) to determine this limit. These semi-waves are solutions of the one-dimensional problem

$$
\begin{cases}u_{t}-d u_{x x}=u[a(x)-b(x) u], & t \in \mathbb{R},-\infty<x<h(t), \\ u(t, h(t))=0, \quad h^{\prime}(t)=-\mu u_{x}(t, h(t)), & t \in \mathbb{R} .\end{cases}
$$

Asymptotic spreading in spatially periodic environment based on Cauchy problem models has received extensive study recently. The spreading speed in such models is usually determined by the so called pulsating fronts, whose existence, uniqueness and other properties have been investigated by many authors; see $[1-5,12,14,16,22]$ and the references therein for more details. In particular, a pulsating front of the reaction diffusion equation

$$
u_{t}-d u_{x x}=u[a(x)-b(x) u], \quad(t, x) \in \mathbb{R}^{2},
$$

is a solution to this equation of the form $u(t, x)=\Psi(x-c t, x)$, where $c$ (the speed) is a positive constant and the function $\Psi(\xi, x)$ (the profile) is $L$-periodic in $x$; moreover, $\lim _{t \rightarrow-\infty} u(t, x)=0, \lim _{t \rightarrow+\infty} u(t, x)$ is the unique 
positive steady state of (1.4). It can be shown that $u(t, x)$ is strictly increasing in $t$. Let us also observe that $u(t+$ $\left.\frac{L}{c}, x\right)=u(t, x-L)$.

By a well-known result of Berestycki, Hamel and Roques [5], there is a minimal speed $c_{*}>0$ such that for each $c \geqslant c_{*},(1.4)$ has a pulsating front with average speed $c$, and no pulsating front exists with average speed $c<c_{*}$. Moreover, it is well known (see $[2,13,22]$ ) that this minimal average speed $c_{*}$ is the spreading speed for the Cauchy problem

$$
v_{t}-d v_{x x}=v[a(x)-b(x) v], \quad x \in \mathbb{R}, t>0 ; \quad v(0, x)=v_{0}(x), \quad x \in \mathbb{R},
$$

where the initial function $v_{0}(x)$ is nonnegative with nonempty compact support.

In contrast to (1.4), we will show that there is only one average speed $C=C(\mu)$ for which (1.3) has a pulsating semi-wave, and such a semi-wave is unique up to translations in $t$ (see Theorem 1.2). Moreover, as mentioned above, this average speed is the spreading speed for the free boundary model (1.1) when spreading happens (Theorem 1.3). Furthermore, we will show that as $\mu \rightarrow \infty, C(\mu)$ increases to $c_{*}$ (Theorem 4.2).

We now describe our results more precisely. Our definition below for pulsating semi-waves to (1.3) is motivated by the notion of pulsating fronts, and ideas in $[18,21]$.

Definition 1.1. We call $(u(t, x), h(t))$ a pulsating semi-wave of (1.3) if it solves (1.3) and

(i) $u(t, x)=U(h(t), h(t)-x)>0$ for $t \in R, x<h(t)$,

(ii) there exists $T>0$ such that $h^{\prime}(t)$ is a positive $T$-periodic function and $h(t+T)-h(t)=L$,

(iii) $U(\tau, \xi) \in C^{1,2}(\mathbb{R} \times[0,+\infty))$ is $L$-periodic in $\tau$.

It will become clear below that $C:=L / T$ is the (average) speed of the semi-wave. Let us also observe that $u(t+T, x)=u(t, x-L)$.

Theorem 1.2. Problem (1.3) always has a pulsating semi-wave $(\tilde{u}, \tilde{h})$. The pulsating semi-wave is unique up to translations in $t$. Furthermore, $\lim _{t \rightarrow \pm \infty} \tilde{h}(t) / t=L / T, \tilde{u}_{t}(t, x)>0$, and $\tilde{u}(t, x) \rightarrow \phi(x)$ as $t \rightarrow+\infty$ uniformly in any interval of the form $(-\infty, M], M \in \mathbb{R}$, where $\phi$ is the unique positive solution of

$$
-d \phi_{x x}=\phi[a(x)-b(x) \phi], \quad x \in \mathbb{R}^{1} .
$$

Note that the existence and uniqueness of $\phi$ is a consequence of Theorem 2.3 of [11]; more general results can be found in $[4,20]$. Using Theorem 1.2, we can deduce the following result on the asymptotic spreading speed determined by (1.1).

Theorem 1.3. Suppose that (1.2) holds, $(u, h)$ is the unique solution of $(1.1)$ and $\lim _{t \rightarrow \infty} h(t)=\infty$; then

$$
\lim _{t \rightarrow \infty} \frac{h(t)}{t}=L / T, \quad \text { where } L / T \text { is the average speed of the semi-wave in Theorem 1.2. }
$$

We remark that Theorem 1.3 only gives the asymptotic speed of the free boundary $|x|=h(t)$. However, from its proof, one sees that for any $\sigma \in(0, \underline{a} / \bar{b})$, where $\underline{a}=\min _{r} a(r)$ and $\bar{a}=\max _{r} b(r)$, the set $\{x: u(x, t)>\sigma\}$ expands to $\mathbb{R}^{N}$ with asymptotic speed $L / T$. Therefore this agrees with the spreading speed in the usual sense.

The existence part of Theorem 1.2 will be proved in Section 2, while Section 3 is devoted to the proof of the rest of Theorem 1.2 as well as some further basic properties of the pulsating semi-wave. The proof of Theorem 1.3 is given in Section 4, where we also show that the spreading speed $L / T$ increases strictly in $\mu$, and as $\mu \rightarrow \infty$, the spreading speed converges to $c_{*}$, which is the minimal speed of the pulsating fronts to (1.4).

This paper is a sequel to [9], where the time-periodic case of the free boundary problem was considered. It turns out that very different techniques have to be used to handle the space-periodic case, though some ideas in the timeperiodic case can be borrowed. Similarly to the approach in [9], we prove the existence of a pulsating semi-wave via a fixed point argument. However, the techniques here are completely new. The proof for uniqueness of the pulsating semi-wave is based on ideas introduced in [9], with considerable changes in the arguments. In [23], independently, (1.3) and its corresponding initial value problem are investigated by a completely different method, which is based on the approach developed in [12]. 
Our analysis in this paper carries over easily when the logistic nonlinearity $u[\alpha(r)-\beta(r) u]$ is replaced by a more general Fisher-KPP type function $f(r, u)$, which is smooth and satisfies

(i) $f(r, 0) \equiv 0, f(r, q(r)) \equiv 0$, where $q(r)$ is bounded from above and below by positive constants,

(ii) $\lim _{r \rightarrow \infty}[f(r, u)-g(r, u)]=0$ locally uniformly in $u \in[0, \infty)$, with $g$ periodic in $r$,

(iii) $f(r, u) / u$ is strictly decreasing in $u$, for every $r \geqslant 0$.

With care, one could further extend the results to the case that $d \Delta u$ is replaced by $\operatorname{div}(d(|x|) \nabla u)$, and $\mu$ is replaced by $\mu(r)$, with suitable conditions on $d(r)$ and $\mu(r)$.

\section{Existence of pulsating semi-waves}

We use $C_{L}^{v}(\mathbb{R})$ to denote the set of all $L$-periodic $C^{v}$ functions and suppose that $p, a, b \in C_{L}^{v}(\mathbb{R})$ for some $v \in$ $(0,1)$, with both $a$ and $b$ positive. In order to prove the existence of a pulsating semi-wave, we consider the following problem

$$
\begin{cases}p(\tau)\left(U_{\tau}+U_{\xi}\right)-d U_{\xi \xi}=U[a(\tau-\xi)-b(\tau-\xi) U], & (\tau, \xi) \in \mathbb{R} \times(0, \infty), \\ U(\tau, 0)=0, & \tau \in \mathbb{R} .\end{cases}
$$

The relationship between (2.1) and (1.3) is given in the following result.

Proposition 2.1. If (2.1) has a positive solution $U(\tau, \xi)$ which is L-periodic in $\tau$, and satisfies $\mu U_{\xi}(\tau, 0) \equiv p(\tau)>0$ for $\tau \in \mathbb{R}$, then $(u(t, x), h(t))$ given by

$$
u(t, x)=U(h(t), h(t)-x), \quad t=\int_{0}^{h(t)} \frac{1}{p(\tau)} d \tau
$$

is a pulsating semi-wave to (1.3).

\section{Proof. Clearly}

$$
1=h^{\prime}(t) \frac{1}{p(h(t))}, \quad \text { i.e., } \quad h^{\prime}(t)=p(h(t)) .
$$

We calculate to obtain

$$
\begin{aligned}
u_{t}-d u_{x x} & =h^{\prime}(t)\left(U_{\tau}+U_{\xi}\right)-d U_{\xi \xi} \\
& =p(h(t))\left[U_{\tau}(h(t), h(t)-x)+U_{\xi}(h(t), h(t)-x)\right]-d U_{\xi \xi} \\
& =U[a(x)-b(x) U] \\
& =u[a(x)-b(x) u],
\end{aligned}
$$

and

$$
h^{\prime}(t)=p(h(t))=\mu U_{\xi}(h(t), 0)=-\mu u_{x}(t, h(t)) .
$$

It is evident that $u(t, h(t))=U(h(t), 0)=0$.

It remains to show that $h^{\prime}(t)$ is $T$-periodic for some $T>0$ and $h(T)-h(0)=L$. We prove that $h^{\prime}(t)$ is $T$-periodic with

$$
T:=\int_{0}^{L} \frac{d s}{p(s)} .
$$

Indeed, from

$$
t=\int_{0}^{h(t)} \frac{d s}{p(s)} \text { and } \quad t+T=\int_{0}^{h(t+T)} \frac{d s}{p(s)}
$$


we obtain

$$
T=\int_{h(t)}^{h(t+T)} \frac{d s}{p(s)}
$$

Since $p(\tau)$ is $L$-periodic and positive, we have

$$
T=\int_{0}^{L} \frac{d s}{p(s)}=\int_{h(t)}^{h(t)+L} \frac{d s}{p(s)} .
$$

Hence

$$
\int_{h(t)}^{h(t+T)} \frac{d s}{p(s)}=\int_{h(t)}^{h(t)+L} \frac{d s}{p(s)}
$$

which implies that

$$
h(t+T)=h(t)+L, \quad h^{\prime}(t+T)=h^{\prime}(t) .
$$

Thus $(u(t, x), h(t))$ is indeed a pulsating semi-wave of (1.3).

Let us note that the pulsating semi-wave given by (2.2) satisfies $h(0)=0$. It is easily seen that for any fixed $t_{0} \in \mathbb{R}$, $\left(u\left(t+t_{0}, x\right), h\left(t+t_{0}\right)\right)$ is also a pulsating semi-wave to (1.3). It will be shown that the pulsating semi-wave is unique subject to this kind of time shifts.

To prove the existence of a function pair $(p(\tau), U(\tau, \xi))$ such that $(2.1)$ holds and $p(\tau)=\mu U_{\xi}(\tau, 0)$, we break the argument into two major steps. In step one, we show that for any given positive $p \in C_{L}^{v}(\mathbb{R}),(2.1)$ has a unique maximal nonnegative solution $U^{p}$ which is $L$-periodic in $\tau$. This defines a mapping $T: p \rightarrow \mu U_{\xi}^{p}(\cdot, 0)$. In the second step, we show that $T$ has a fixed point $p^{*}$, and thus obtain the required solution pair $\left(p^{*}, U^{p^{*}}\right)$.

In order to apply suitable fixed point theorems to the operator $T$, it is convenient to consider nonnegative $p$, but $h(t)$ is not well defined for such $p$. To avoid this difficulty, we use a perturbation approach. For small $\epsilon>0$, we replace the original problem $(2.1)$ by

$$
\begin{cases}p_{\epsilon}(\tau)\left(U_{\tau}+U_{\xi}\right)-d U_{\xi \xi}=U[a(\tau-\xi)-b(\tau-\xi) U], & (\tau, \xi) \in \mathbb{R} \times(0,+\infty), \\ U(\tau, 0)=0, & \tau \in \mathbb{R},\end{cases}
$$

where

$$
p_{\epsilon}(\tau)=\max \{p(\tau), \epsilon\} .
$$

We will show that (2.3) has a unique maximal nonnegative solution $U^{p, \epsilon}(\tau, \xi)$ that is $L$-periodic in $\tau$, and the operator $T^{\epsilon}: p \rightarrow \mu U_{\xi}^{p, \epsilon}(\cdot, 0)$ has a fixed point $p^{\epsilon}$. Moreover, we will show that there exists $\delta>0$ such that $p^{\epsilon} \geqslant \delta$ for all small $\epsilon$. Hence $\left(p^{\epsilon}, U^{p^{\epsilon}, \epsilon}\right)$ solves the original unperturbed problem when $\epsilon \in(0, \delta]$, and $U^{p^{\epsilon}, \epsilon}$ is a positive solution.

\subsection{Existence of $U^{p, \epsilon}$}

In this subsection we show that for any $p \in C_{L}^{\nu}(\mathbb{R})$ and $\epsilon>0,(2.3)$ has a maximal nonnegative solution $U^{p, \epsilon}$. We also determine exactly when this solution is positive. To this end, we need the following eigenvalue problem

$$
\left\{\begin{array}{l}
\mathcal{L}_{\epsilon} U=\lambda a(\tau-\xi) U, \quad(\tau, \xi) \in \mathbb{R}^{2} \\
\text { where } \mathcal{L}_{\epsilon} U=p_{\epsilon}(\tau)\left(U_{\tau}+U_{\xi}\right)-d U_{\xi \xi} .
\end{array}\right.
$$

Proposition 2.2. The following conclusions hold: 
(1) For any nonnegative $p \in C_{L}^{v}(\mathbb{R})$, and $\epsilon>0, \alpha \in \mathbb{R}$, there exists a unique $\lambda(\alpha, p, \epsilon) \in \mathbb{R}$ with corresponding positive function $\phi_{\alpha, p, \epsilon}=\phi_{\alpha, p, \epsilon}(\tau, \xi) \in C^{1,2}(\mathbb{R} \times \mathbb{R})$, which is L-periodic both in $\tau$ and $\xi$, such that $(\lambda, U)=$ $\left(\lambda(\alpha, p, \epsilon), e^{\alpha \xi} \phi_{\alpha, p, \epsilon}\right)$ satisfies (2.4).

(2) Let

$$
\begin{aligned}
\lambda_{1}(p, \epsilon)= & \sup \left\{\lambda \in \mathbb{R}: \exists \phi \in C^{1,2}(\mathbb{R} \times \mathbb{R}), \phi(\tau, \xi)\right. \text { is positive } \\
& \text { and L-periodic in } \left.\tau, \text { and } \mathcal{L}_{\epsilon} \phi \geqslant \lambda a(\tau-\xi) \phi\right\} .
\end{aligned}
$$

Then there is a unique $\alpha_{0} \in \mathbb{R}$ such that $\lambda_{1}(p, \epsilon)=\lambda\left(\alpha_{0}, p, \epsilon\right)$. Moreover, $\alpha_{0}>0$.

(3) Suppose $\lambda=\tilde{\lambda}(l, p, \epsilon)$ is the principal eigenvalue of the eigenvalue problem

$$
\left\{\begin{array}{l}
\mathcal{L}_{\epsilon} U=\lambda a(\tau-\xi) U, \quad(\tau, \xi) \in \mathbb{R} \times(0, l), \\
U(\tau, \xi)=U(\tau+L, \xi), \quad U(\tau, 0)=U(\tau, l)=0 .
\end{array}\right.
$$

Then $\tilde{\lambda}(l, p, \epsilon)$ decreases to $\lambda_{1}(p, \epsilon)$ as $l \rightarrow+\infty$.

(4) $\tilde{\lambda}(l, p, \epsilon)$ is continuous in $(l, p, \epsilon)$ and $\lambda(\alpha, p, \epsilon)$ is continuous in $(\alpha, p, \epsilon)$, where $l, \epsilon>0, \alpha \in \mathbb{R}$ and $p \in C_{L}^{\nu}(\mathbb{R})$.

(5) $\lambda(\alpha, p, \epsilon)$ is concave in $\alpha$.

Proof. All the conclusions here follow from the main results of [19], except that we need to prove that $\alpha_{0}>0$ in conclusion (2). By conclusions (4) and (5), $\lambda(\alpha, p, \epsilon)$ is continuous and concave in $\alpha$. Moreover, it is obvious that $\lambda(0, p, \epsilon)=0$ (with corresponding $\phi_{0, p, \epsilon}$ a positive constant). Hence, we only need to prove that there is some $\alpha^{\prime}>0$ such that $\lambda\left(\alpha^{\prime}, p, \epsilon\right)=0$. To show this, due to the uniqueness of the principal eigenvalue proved in [19], it suffices to find a positive function $\phi_{0}(\tau, \xi)$ which is $L$-periodic in $\tau$, and a positive number $\alpha^{\prime}$, such that $U_{0}:=e^{\alpha^{\prime} \xi} \phi_{0}(\tau, \xi)$ satisfies $\mathcal{L}_{\epsilon} U_{0}=0$. We now look for such a function $\phi_{0}$ of the special form $\phi_{0}(\tau, \xi)=\psi(\tau)$. Then $\mathcal{L}_{\epsilon} U_{0}=0$ reduces to

$$
\left\{p_{\epsilon}(\tau) \psi^{\prime}(\tau)+\left[p_{\epsilon}(\tau) \alpha^{\prime}-d\left(\alpha^{\prime}\right)^{2}\right] \psi(\tau)\right\} e^{\alpha^{\prime} \xi}=0 .
$$

Hence

$$
\psi^{\prime}=\alpha^{\prime}\left[\frac{d}{p_{\epsilon}(\tau)} \alpha^{\prime}-1\right] \psi
$$

Since $\psi(\tau)$ is $L$-periodic, we have

$$
\alpha^{\prime} \int_{0}^{L} \frac{d}{p_{\epsilon}(\tau)} d \tau=L,
$$

which implies $\alpha^{\prime}>0$. Clearly

$$
\psi(\tau)=\exp \int \alpha^{\prime}\left[\frac{d \alpha^{\prime}}{p_{\epsilon}(\tau)}-1\right] d \tau>0
$$

This completes our proof.

We also need the following auxiliary logistic problem

$$
p_{\epsilon}(\tau)\left(U_{\tau}+U_{\xi}\right)-d U_{\xi \xi}=U[a(\tau-\xi)-b(\tau-\xi) U], \quad(\tau, \xi) \in \mathbb{R}^{2} .
$$

The following result is contained in [20].

Proposition 2.3. Problem (2.6) has a positive solution $\bar{U}^{p, \epsilon}=\bar{U}^{p, \epsilon}(\tau, \xi)$ which is L-periodic both in $\tau$ and $\xi$ if and only if $\lambda(0, p, \epsilon)<1$. Moreover, for any nonnegative L-periodic continuous initial function $\psi=\psi(\xi) \not \equiv 0$, the solution of (2.6) with $U(0, \xi)=\psi(\xi)$, denoted by $U(\tau, \xi, \psi)$, satisfies $\lim _{\tau \rightarrow+\infty}\left|U(\tau, \xi, \psi)-\bar{U}^{p, \epsilon}(\tau, \xi)\right|=0$ uniformly for $\xi \in \mathbb{R}$.

Remark 2.4. If $p(\tau) \geqslant \epsilon$ for $\tau \in \mathbb{R}$, then by the transformation in Proposition $2.1, u(t, x)=\bar{U}^{p, \epsilon}(h(t), h(t)-x)$ is a positive entire solution of (1.4), which is $L$-periodic in $x$ and $\int_{0}^{L} 1 / p(\tau) d \tau$-periodic in $t$. Hence it must coincide with $\phi(x)$, the unique positive periodic solution of $-d u_{x x}=u[a(x)-b(x) u], x \in \mathbb{R}$. 
Now, we are ready to show the main result of this subsection.

Proposition 2.5. The following conclusions hold:

(1) For any fixed $p \in C_{L}^{v}(\mathbb{R})$ and any positive constant $\epsilon$, problem (2.3) admits a maximal nonnegative L-periodic in $\tau$ solution $U^{p, \epsilon}=U^{p, \epsilon}(\tau, \xi)$ in the sense that any other nonnegative L-periodic in $\tau$ solution is bounded from above by $U^{p, \epsilon}$. Furthermore, $U^{p, \epsilon} \equiv 0$ if and only if $\lambda_{1}(p, \epsilon) \geqslant 1$.

(2) For any $p \in C_{L}^{\nu}(\mathbb{R})$ and $\epsilon>0$ with $\lambda_{1}(p, \epsilon)<1, U^{p, \epsilon}$ is the unique positive L-periodic in $\tau$ solution of (2.3). Moreover, $\lim _{\xi \rightarrow+\infty}\left|U^{p, \epsilon}(\tau, \xi)-\bar{U}^{p, \epsilon}(\tau, \xi)\right|=0$ uniformly for $\tau \in \mathbb{R}$.

Proof. First, we make use of some simple facts whose easy proof can be found in Step 1 of the proof of Proposition 2.1 in [9]. Suppose that $u^{l}$ is the unique positive solution of

$$
\left\{\begin{array}{l}
-d u_{\xi \xi}=u(\bar{a}-\underline{b} u) \quad \text { in }(0, \ell), \\
u(0)=0, \quad u(\ell)=\infty
\end{array}\right.
$$

where

$$
\bar{a}=\max _{r} a(r), \quad \underline{b}=\min _{r} b(r),
$$

and $u^{\infty}$ is the limit of $u^{l}$ as $l \rightarrow+\infty$. Then $u^{\infty}=u^{\infty}(\xi) \leqslant \max a / \min b$, and for every constant $M \geqslant 1, M u^{\infty}$ is a supersolution of (2.3). By a sweeping argument, any $L$-periodic in $\tau$ solution of (2.3) is bounded from above by $\sup u^{\infty}$.

Let us now prove conclusion (1). When $\lambda_{1}(p, \epsilon) \geqslant 1$, by Proposition 2.2, there is some $\alpha_{0}>0$ such that $\lambda_{1}(p, \epsilon)=$ $\lambda\left(\alpha_{0}, p, \epsilon\right)$. It is easily seen that the corresponding function $e^{\alpha_{0} \xi} \phi_{\alpha_{0}, p, \epsilon}$ and its product with any positive constant are $L$-periodic in $\tau$ supersolutions of (2.3). Suppose that (2.3) has a positive $L$-periodic in $\tau$ solution $U$. Then by the boundedness of $U$, we can suppose that $M e^{\alpha_{0} \xi} \phi_{\alpha_{0}, p, \epsilon}(\tau, \xi) \geqslant U(\tau, \xi)$ on $\mathbb{R} \times[0,+\infty)$. We may assume that $M>0$ is the minimal constant such that the above inequality holds. Since $\alpha_{0}>0$, the equality must hold at some $\left(\tau_{0}, \xi_{0}\right)$ in the above inequality. But the strong maximum principle shows that it is impossible. Hence the maximal nonnegative $L$-periodic solution $U^{p, \epsilon}$ is identically 0 when $\lambda_{1}(p, \epsilon) \geqslant 1$.

In the remaining case $\lambda_{1}(p, \epsilon)<1$, by Proposition 2.2, there is some large $l$ such that $\tilde{\lambda}(l, p, \epsilon)<1$. Suppose that $\tilde{\phi}_{l, p, \epsilon}>0$ is the corresponding eigenfunction of (2.5) normalized by $\max \tilde{\phi}_{l, p, \epsilon}=1$. Then for any sufficiently small constant $\gamma$,

$$
\psi(\tau, \xi)= \begin{cases}\gamma \tilde{\phi}_{l, p, \epsilon}(\tau, \xi-l), & \tau \in \mathbb{R}, \xi \in[l, 2 l], \\ 0, & \tau \in \mathbb{R}, \xi \in \mathbb{R} \backslash[l, 2 l]\end{cases}
$$

is a subsolution of (2.3). Therefore, we can find a maximal positive $L$-periodic in $\tau$ solution $U^{p, \epsilon}$ in the order interval $\left[\psi, u^{\infty}\right]$. Since any positive solution of (2.3) is bounded from above by $u^{\infty}, U^{p, \epsilon}$ is the maximal positive solution.

Finally we prove conclusion (2). Let $\psi(\tau, \xi)$ be defined as above, with $l=k L$ for some large positive integer $k$, so that it is an $L$-periodic in $\tau$ subsolution of (2.3) for small $\gamma$. For each nonnegative integer $i$ we define

$$
\psi_{i}(\tau, \xi)=\psi(\tau, \xi-i k L)
$$

It is easily seen that $\psi_{i}$ is a subsolution of (2.3). Let $U=U(\tau, \xi)$ be a positive $L$-periodic in $\tau$ solution of (2.3). For each $i \geqslant 0$, there exists $\sigma_{i} \in(0,1)$ such that $U(\tau, \xi)>\sigma_{i} \psi_{i}(\tau, \xi)$ for $\tau \in \mathbb{R}$ and $\xi \geqslant 0$. For any $\sigma \in\left[\sigma_{i}, 1\right], \sigma \psi_{i}$ is a subsolution of (2.3). We claim that $U(\tau, \xi)>\sigma \psi_{i}(\tau, \xi)$ for all $\tau \in \mathbb{R}, \xi \geqslant 0$ and $\sigma \in\left[\sigma_{i}, 1\right]$. Otherwise, there exists $\sigma_{*} \in\left(\sigma_{i}, 1\right]$ and $\left(\tau_{*}, \xi_{*}\right) \in(0, L] \times((i+1) k L,(i+2) k L)$ such that

$$
U(\tau, \xi) \geqslant \sigma_{*} \psi_{i}(\tau, \xi) \quad \text { for } \tau \in \mathbb{R}, \xi \geqslant 0 ; \quad U\left(\tau_{*}, \xi_{*}\right)=\sigma_{*} \psi_{i}\left(\tau_{*}, \xi_{*}\right) .
$$

We may then apply the strong maximum principle to conclude that $U \equiv \sigma_{*} \psi_{i}$, a contradiction. This proves the claim, which implies in particular,

$$
U(\tau, \xi)>\psi_{i}(\tau, \xi) \quad \text { for } \tau \in \mathbb{R}, \xi \geqslant 0 .
$$

Let $\left\{\xi_{i}\right\}$ be an arbitrary sequence increasing to $+\infty$ as $i \rightarrow+\infty$, and define $U_{i}(\tau, \xi)=U\left(\tau, \xi+\xi_{i}\right)$. By applying standard $L^{p}$ theory (see [17]) to the equation satisfied by $U_{i}$ and Sobolev embedding (see Lemma 3.3 in [15]), one sees 
that by passing to a subsequence, $U_{i} \rightarrow \check{U}$ as $i \rightarrow \infty$ locally uniformly in $\mathbb{R}^{2}$, and $\check{U}$ is $L$-periodic in $\tau$. Moreover, if we write $\xi_{i}=n_{i} L+\tilde{\xi}_{i}$ with $\tilde{\xi}_{i} \in[0, L)$, and assume that $\tilde{\xi}_{i} \rightarrow \tilde{\xi}_{0}$, then $\check{U}\left(\tau, \xi-\tilde{\xi}_{0}\right)$ solves (2.6). Furthermore, due to (2.8), we always have $\sup _{(\tau, \xi) \in \mathbb{R}^{2}} \check{U}\left(\tau, \xi-\tilde{\xi}_{0}\right) \geqslant \gamma$. Therefore, $\check{U}\left(\tau, \xi-\tilde{\xi}_{0}\right)$ is a positive $L$-periodic in $\tau$ solution of (2.6). Hence $\check{U}\left(\tau, \xi-\widetilde{\xi}_{0}\right) \equiv \bar{U}^{p, \epsilon}(\tau, \xi)$. Clearly the above discussion implies that $\lim _{\xi \rightarrow+\infty} \mid U(\tau, \xi)-$ $\bar{U}^{p, \epsilon}(\tau, \xi) \mid=0$ uniformly for $\tau \in \mathbb{R}$.

It remains to show the uniqueness of $U$. We follow a standard argument. If there is another positive $L$-periodic in $\tau$ solution $\tilde{U} \not \equiv U$, then from the conclusion proved above, we have

$$
\lim _{\xi \rightarrow+\infty} \frac{U(\tau, \xi)}{\tilde{U}(\tau, \xi)}=1 \quad \text { uniformly in } \tau \in \mathbb{R} .
$$

Moreover, by the Hopf boundary lemma, $U_{\xi}(\tau, 0)$ and $\tilde{U}_{\xi}(\tau, 0)$ are bounded away from 0 and $\infty$ for all $\tau \in \mathbb{R}$. Therefore there is some $M \in(0,1)$ such that $\tilde{U}(\tau, \xi) \geqslant M U(\tau, \xi)$ on $\mathbb{R} \times[0,+\infty)$, and we may assume that $M$ takes the maximal value such that this inequality holds (we may interchange $U$ and $\tilde{U}$ to guarantee $M<1$ ). Due to (2.9), either $\tilde{U}\left(\tau_{0}, \xi_{0}\right)=M U\left(\tau_{0}, \xi_{0}\right)$ at some $\tau_{0} \in \mathbb{R}, \xi_{0} \in(0,+\infty)$ or $\frac{\partial \tilde{U}}{\partial \xi}\left(\tau_{0}, 0\right)=M \frac{\partial U}{\partial \xi}\left(\tau_{0}, 0\right)$, and the strong maximum principle then implies $\tilde{U} \equiv M U$. But $M<1$ implies that $M U$ is not a solution of (2.3). This contradiction completes our proof.

We may now define the mapping $T^{\epsilon}$ on $C_{L}^{v}(\mathbb{R})$ by $T^{\epsilon}(p)=\mu U_{\xi}^{p, \epsilon}(\cdot, 0)$.

\section{2. $T^{\epsilon}$ has a fixed point}

We are going to use Schauder's fixed point theorem to conclude that $T^{\epsilon}$ has a fixed point.

Lemma 2.6. $T^{\epsilon}$ is completely continuous on $C_{L}^{v}(\mathbb{R})$.

Proof. First, we show that for any positive $\delta \in(0,1)$, the norm of $U_{\xi}^{p, \epsilon}(\cdot, 0)$ in $C_{L}^{\delta}(\mathbb{R})$ can be controlled by $\|p\|_{C_{L}^{0}(\mathbb{R})}$ when all other parameters are fixed.

Set

$$
s=f(\tau):=\int_{0}^{\tau} \beta_{\epsilon}(t) d t, \quad V(s, \xi):=U^{p, \epsilon}\left(f^{-1}(s), \xi\right)
$$

where $\beta_{\epsilon}(t)=\frac{1}{p_{\epsilon}(t)}$. Then $V(s, \xi)$ is periodic in $s$ with period $\int_{0}^{L} \beta_{\epsilon}(t) d t$, and is a positive solution of

$$
\begin{cases}V_{s}+p_{\epsilon}\left(f^{-1}(s)\right) V_{\xi}-d V_{\xi \xi}=V\left[a\left(f^{-1}(s)-\xi\right)-b\left(f^{-1}(s)-\xi\right) V\right], & s \in \mathbb{R}, \xi>0, \\ V(s, 0)=0, & s \in \mathbb{R} .\end{cases}
$$

Since $0 \leqslant V \leqslant u^{\infty} \leqslant \max a / \min b$, we can apply the $L^{p}$ estimates (see, for example, Theorem 7.15 of [17]) to (2.10) to conclude that $\left\|V\left(s_{0}+\cdot, \cdot\right)\right\|_{W_{q}^{1,2}([0,1] \times[0, l])} \leqslant C$ for all $s_{0} \in \mathbb{R}, q>1, l>0$ and some constant $C$ depending only on $\|p\|_{C_{L}^{0}(\mathbb{R})}, l$ and $q$. By Sobolev embedding (see, e.g. Lemma 3.3 of $[15]$ ), we obtain, for every $\delta \in(0,1)$,

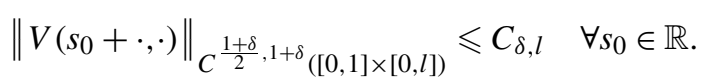

Therefore, we have

$$
\begin{aligned}
\frac{\left|U_{\xi}^{p, \epsilon}\left(t_{1}, 0\right)-U_{\xi}^{p, \epsilon}\left(t_{2}, 0\right)\right|}{\left|t_{1}-t_{2}\right|^{\delta}} & =\frac{\left|V_{\xi}\left(f\left(t_{1}\right), 0\right)-V_{\xi}\left(f\left(t_{2}\right), 0\right)\right|}{\left|f\left(t_{1}\right)-f\left(t_{2}\right)\right|^{\delta}} \cdot \frac{\left|f\left(t_{1}\right)-f\left(t_{2}\right)\right|^{\delta}}{\left|t_{1}-t_{2}\right|^{\delta}} \\
& \leqslant C\left|\beta_{\epsilon}(\cdot)\right|_{C_{L}^{0}(\mathbb{R})}^{\delta} \leqslant C \epsilon^{-\delta} .
\end{aligned}
$$

This implies that, for any $M>0,\left\{U_{\xi}^{p, \epsilon}(\cdot, 0):\|p\|_{C_{L}^{v}(\mathbb{R})} \leqslant M\right\}$ is bounded in $C_{L}^{\delta}(\mathbb{R})$ for some $\delta \in(\nu, 1)$, and hence it is pre-compact in $C_{L}^{v}(\mathbb{R})$. This proves that $T^{\epsilon}$ maps any bounded set of $C_{L}^{v}(\mathbb{R})$ into a pre-compact set in this space. 
Moreover, if $p_{n} \rightarrow p_{0}$ in $C_{L}^{v}(\mathbb{R})$, then by compactness, by passing to a subsequence, $U^{p_{n}, \epsilon}(t, x) \rightarrow \tilde{U}^{p_{0}, \epsilon}(t, x)$ in $C_{\text {loc }}^{\frac{1+v}{2}, 1+v}(\mathbb{R} \times[0,+\infty))$, where $\tilde{U}^{p, \epsilon}$ is some nonnegative $L$-periodic in $\tau$ solution of (2.3) with $p=p_{0}$. It follows that $U_{\xi}^{p_{n}, \epsilon}(\cdot, 0) \rightarrow \tilde{U}_{\xi}^{p_{0}, \epsilon}(\cdot, 0)$ in $C_{L}^{v}(\mathbb{R})$. What we need to show is that $\tilde{U}^{p_{0}, \epsilon}$ is just $U^{p_{0}, \epsilon}$. If $\lambda_{1}\left(p_{0}, \epsilon\right) \geqslant 1$, then 0 is the unique nonnegative $L$-periodic in $\tau$ solution of (2.3), and our conclusion holds. If $\lambda_{1}\left(p_{0}, \epsilon\right)<1$, we can find a sufficiently small constant $\gamma$ independent of $p_{n}$ such that

$$
\psi^{n}(\tau, \xi)= \begin{cases}\gamma \tilde{\phi}_{l, p_{n}, \epsilon}(\tau, \xi), & \tau \in \mathbb{R}, \xi \in[0, l], \\ 0, & \tau \in \mathbb{R}, \xi \in(l,+\infty)\end{cases}
$$

is a subsolution of (2.3) with $p=p_{n}$, where $\left\|\tilde{\phi}_{l, p_{n}, \epsilon}\right\|_{C^{0}(\mathbb{R} \times[0, l])}=1$. It follows that $U^{p_{n}, \epsilon} \geqslant \psi^{n}$ and hence $\left\|U^{p_{n}, \epsilon}\right\|_{C^{0}(\mathbb{R} \times[0,+\infty))} \geqslant \gamma>0$, which infers $\left\|\tilde{U}^{p_{0}, \epsilon}\right\|_{C^{0}(\mathbb{R} \times[0,+\infty))}>0$. Thus $\tilde{U}^{p_{0}, \epsilon} \equiv U^{p_{0}, \epsilon}$. This shows that $T^{\epsilon}$ is continuous.

In order to use Schauder's fixed point theorem to prove the existence of a fixed point of $T^{\epsilon}$, we look for an invariant set of $T^{\epsilon}$ which is bounded, closed and convex. In the proof of Proposition 2.5, we have shown that $U^{p, \epsilon}(\tau, \xi) \leqslant$ $u^{\infty}(\xi)$ on $\mathbb{R} \times[0,+\infty)$ for any $p \in C_{L}^{v}(\mathbb{R})$. We note that $U^{p, \epsilon}(\tau, 0)=u^{\infty}(0)=0$.

Lemma 2.7. There exists $M=M_{\epsilon}>0$ such that $E_{\epsilon}:=\left\{p \in C_{L}^{v}(\mathbb{R}):\|p\|_{C_{L}^{v}(\mathbb{R})} \leqslant M, 0 \leqslant p(\tau) \leqslant \mu\left(u^{\infty}\right)^{\prime}(0) \forall \tau \in \mathbb{R}\right\}$ is an invariant set of $T^{\epsilon}$.

Proof. First, for any $p \in C_{L}^{v}(\mathbb{R}), 0 \leqslant U_{\xi}^{p, \epsilon}(\tau, 0) \leqslant\left(u^{\infty}\right)^{\prime}(0)$. Moreover, for $p(\cdot) \in \tilde{E}:=\left\{p \in C_{L}^{v}(\mathbb{R}): 0 \leqslant p(\tau) \leqslant\right.$ $\left.\mu\left(u^{\infty}\right)^{\prime}(0) \forall \tau \in \mathbb{R}\right\},\|p\|_{\infty} \leqslant \mu\left(u^{\infty}\right)^{\prime}(0)$, and from (2.3) we see, by standard $L^{p}$ theory for parabolic equations (see [17]) that $\left\{U^{p, \epsilon}\right\}$ has a bound in $W_{q}^{1,2}([0, L] \times[0,1])(\forall q>1)$ that is independent of $p \in \tilde{E}$. By Sobolev embedding we know that $\left\{U^{p, \epsilon}\right\}$ has a bound in $C^{\frac{1+v}{2}, 1+v}([0, L] \times[0,1])(v \in(0,1))$ that is independent of $p \in \tilde{E}$. Therefore $\left\{U_{\xi}^{p, \epsilon}(\cdot, 0): p \in \tilde{E}\right\}$ is bounded in $C_{L}^{v}(\mathbb{R})$, say $\left\|U_{\xi}^{p, \epsilon}(\cdot, 0)\right\|_{C_{L}^{v}(\mathbb{R})} \leqslant M_{\epsilon}$ for all $p \in \tilde{E}$. Define $E_{\epsilon}:=\{p \in$ $\left.\tilde{E}:\|p\|_{C_{L}^{v}(\mathbb{R})} \leqslant M_{\epsilon}\right\}$. Then clearly $E_{\epsilon}$ is invariant under $T^{\epsilon}$.

Thus by Schauder's fixed point theorem we obtain

Proposition 2.8. For any $\epsilon>0, T^{\epsilon}$ has a fixed point $\bar{p}^{\epsilon}$ in $E_{\epsilon}$.

2.3. $\bar{p}^{\epsilon}(\tau) \geqslant \epsilon$ for all small $\epsilon>0$

Proposition 2.9. There is some $\epsilon_{0}>0$ such that $\bar{p}^{\epsilon_{0}}(\tau)>\epsilon_{0}$ on $\mathbb{R}$ and hence for $p=\bar{p}^{\epsilon_{0}}$, (2.1) has a positive L-periodic in $\tau$ solution $U^{\bar{p}^{\epsilon_{0}}, \epsilon_{0}}$ such that $\bar{p}^{\epsilon_{0}}(\tau)=\mu U_{\xi}^{\bar{p}^{\epsilon_{0}}, \epsilon_{0}}(\tau, 0)$.

Proof. We only need to show that $\liminf _{\epsilon \rightarrow 0} \min _{\tau \in \mathbb{R}} \bar{p}^{\epsilon}(\tau)>0$. We use two steps to prove this conclusion.

Step 1. We show that $\liminf _{\epsilon \rightarrow 0}\left\|\bar{p}^{\epsilon}\right\|_{C_{L}^{0}(\mathbb{R})}>0$.

Recall that

$$
\underline{a}=\min a, \quad \bar{a}=\max a, \quad \underline{b}=\min b, \quad \bar{b}=\max b .
$$

First, we can find some $\delta_{0}$ small and $l_{0}>0$ large such that for each $\delta \in\left(0, \delta_{0}\right]$ and $l \geqslant l_{0}$, the problem

$$
-d U^{\prime \prime}+\delta U^{\prime}=U[\underline{a}-\bar{b} U] \quad \text { in }(0, l), U(0)=U^{\prime}(l)=0
$$

has a unique positive solution $U_{l}$, and $U_{l}$ satisfies $U_{l}^{\prime}(\xi)>0$ in $[0, l)$. (The existence follows from a simple upper and lower solution argument, and the uniqueness is a consequence of the concavity of the nonlinearity. The fact that $U_{l}^{\prime}>0$ in $[0, l)$ follows from $U_{l}\left(\underline{a}-\bar{b} U_{l}\right)>0$ in $[0, l)$ and $U_{l}^{\prime}(l)=0$.)

Define $\underline{U}(\xi)=U_{l}(\xi)$ in $[0, l]$ and $\underline{U}(\xi)=U_{l}(2 l-\xi)$ for $\xi \in(l, 2 l]$, and $\delta(\xi)=\delta \operatorname{sgn}(l-\xi)$. Then it is easily seen that $\underline{U}$ is a weak solution of

$$
-d U^{\prime \prime}+\delta(\xi) U^{\prime}=U(\underline{a}-\bar{b} U) \quad \text { in }(0,2 l), U(0)=U(2 l)=0 .
$$


If $p(\tau)<\delta$ in $\mathbb{R}$, then clearly $p(\tau) \underline{U}^{\prime}(\xi) \leqslant \delta(\xi) \underline{U}^{\prime}(\xi)$ in $[0,2 l]$ for all $\tau$. It follows easily from the comparison principle that $U^{p, \epsilon}(\tau, \xi) \geqslant \underline{U}(\xi)$ for all $\tau \in \mathbb{R}$ and $\xi \in[0,2 l]$. Hence $U_{\xi}^{p, \epsilon}(\tau, 0) \geqslant \underline{U}^{\prime}(0)$ for all $\tau$.

If $\liminf _{\epsilon \rightarrow 0}\left\|\bar{p}^{\epsilon}\right\|_{C_{L}^{0}(\mathbb{R})}=0$, then we can find some $\epsilon_{0}<\min \left\{\delta, \underline{U}^{\prime}(0)\right\}$ such that $\bar{p}^{\epsilon_{0}}(\tau)<\min \left\{\delta, \mu \underline{U}^{\prime}(0)\right\}$ for all $\tau$. This and $U^{\bar{p}^{\epsilon_{0}}, \epsilon_{0}}(\tau, \xi) \geqslant \underline{U}(\xi)$ on $\mathbb{R} \times[0,+\infty)$ imply that $\mu U_{\xi}^{\bar{p}^{\epsilon_{0}}, \epsilon_{0}}(\tau, 0) \geqslant \mu \underline{U}^{\prime}(0)>\bar{p}^{\epsilon_{0}}(\tau)$, contradicting $\bar{p}^{\epsilon_{0}}(\tau)=\mu U_{\xi}^{\bar{p}^{\epsilon_{0}}, \epsilon_{0}}(\tau, 0)$.

Step 2. We show that $\liminf _{\epsilon \rightarrow 0} \min _{\tau \in \mathbb{R}} \bar{p}^{\epsilon}(\tau)>0$. Suppose by way of contradiction that this limit is 0 . We are going to derive a contradiction. Since $2 \theta:=\liminf _{\epsilon \rightarrow 0}\left\|\bar{p}^{\epsilon}\right\|_{C_{L}^{v}(\mathbb{R})}>0$, for every small $\epsilon>0$, there is some $\tau_{\epsilon} \in[0, L]$ such that $\bar{p}^{\epsilon}\left(\tau_{\epsilon}\right)=\theta$. Set

$$
s=f_{\epsilon}(\tau):=\int_{\tau_{\epsilon}}^{\tau} \beta_{\epsilon}(t) d t \quad \text { with } \beta_{\epsilon}(t)=\frac{1}{\max \left\{\bar{p}^{\epsilon}(t), \epsilon\right\}} .
$$

It is easy to check that $f_{\epsilon}^{-1}(0)=\tau_{\epsilon}, \bar{p}^{\epsilon}\left(f_{\epsilon}^{-1}(0)\right)=\theta$, and if the function $g(\tau)$ is $L$-periodic then $g\left(f_{\epsilon}^{-1}(s)\right)$ is periodic with period $S_{\epsilon}:=\int_{0}^{L} \beta_{\epsilon}(t) d t$.

Denote

$$
V^{\epsilon}(s, \xi):=U^{\bar{p}^{\epsilon}, \epsilon}\left(f_{\epsilon}^{-1}(s), \xi\right), \quad q^{\epsilon}(s)=\bar{p}^{\epsilon}\left(f_{\epsilon}^{-1}(s)\right),
$$

and

$$
\eta_{1}^{\epsilon}(s, \xi)=a\left(f_{\epsilon}^{-1}(s)-\xi\right), \quad \eta_{2}^{\epsilon}(s, \xi)=b\left(f_{\epsilon}^{-1}(s)-\xi\right) .
$$

Then $V^{\epsilon}(s, \xi)$ is a positive solution of

$$
\begin{cases}U_{s}+\max \left\{q^{\epsilon}(s), \epsilon\right\} U_{\xi}-d U_{\xi \xi}=U\left[\eta_{1}^{\epsilon}(s, \xi)-\eta_{2}^{\epsilon}(s, \xi) U\right], & (s, \xi) \in \mathbb{R} \times(0, \infty), \\ U(s, 0)=0, \quad U(s, \xi)=U\left(s+S_{\epsilon}, \xi\right), & (s, \xi) \in \mathbb{R} \times(0, \infty) .\end{cases}
$$

Moreover,

$$
q^{\epsilon}(s)=\mu V_{\xi}^{\epsilon}(s, 0) \quad \forall s \in \mathbb{R} .
$$

Let $\epsilon_{n} \rightarrow 0$ be such that $\min _{\tau \in \mathbb{R}} \bar{p}_{\epsilon_{n}}(\tau) \rightarrow 0$. Then from

$$
0 \leqslant q^{\epsilon_{n}} \leqslant \mu\left(u^{\infty}\right)^{\prime}(0), \quad \underline{a} \leqslant \eta_{1}^{\epsilon_{n}} \leqslant \bar{a}, \quad \underline{b} \leqslant \eta_{2}^{\epsilon_{n}} \leqslant \bar{b}
$$

we see that, by passing to a subsequence,

$q^{\epsilon_{n}} \rightarrow q \quad$ weakly in $L^{2}\left(K_{1}\right), \quad \eta_{1}^{\epsilon_{n}} \rightarrow \eta_{1} \quad$ and $\quad \eta_{2}^{\epsilon_{n}} \rightarrow \eta_{2} \quad$ weakly in $L^{2}\left(K_{1} \times K_{2}\right)$,

for every compact set $K_{1} \subset \mathbb{R}$ and every compact set $K_{2} \subset[0, \infty)$. Clearly

$$
0 \leqslant q \leqslant \mu\left(u^{\infty}\right)^{\prime}(0), \quad \underline{a} \leqslant \eta_{1} \leqslant \bar{a}, \quad \underline{b} \leqslant \eta_{2} \leqslant \bar{b} .
$$

Moreover, applying $L^{p}$ estimates to the equation of $V^{\epsilon_{n}}$ we find that $\left\{V^{\epsilon_{n}}\right\}$ is bounded in $W_{p}^{1,2}\left(K_{1} \times K_{2}\right)(\forall p>1)$ with compact sets $K_{1}$ and $K_{2}$ as described above. Therefore by Sobolev embedding we may assume, by passing to a subsequence, that $V^{\epsilon_{n}} \rightarrow V$ in $C_{l o c}^{\frac{1+v}{2}}, 1+v(\mathbb{R} \times[0,+\infty))$ with $0<v<1$. It follows that $q^{\epsilon}(s)=\mu V_{\xi}^{\epsilon}(s, 0)$ converges to $q(s)$ in $C_{\text {loc }}^{v}(\mathbb{R})$ along $\epsilon=\epsilon_{n}$. Letting $n \rightarrow \infty$ in the equation for $V^{\epsilon_{n}}$ we find that $V$ is a nonnegative weak solution of

$$
\begin{cases}V_{s}+q(s) V_{\xi}-d V_{\xi \xi}=V\left[\eta_{1}(s, \xi)-\eta_{2}(s, \xi) V\right], & (s, \xi) \in \mathbb{R} \times(0, \infty), \\ V(s, 0)=0, & s \in \mathbb{R} .\end{cases}
$$

We also have

$$
q(s)=\mu V_{\xi}(s, 0), \quad 0 \leqslant V(s, \xi) \leqslant u^{\infty}(\xi) \leqslant \bar{a} / \underline{b} \forall s \in \mathbb{R}, \forall \xi \geqslant 0 .
$$

Since $V_{\xi}^{\epsilon}(0,0)=\bar{p}^{\epsilon}\left(f^{-1}(0)\right)=\theta$, we have $V_{\xi}(0,0)=\theta>0$. Therefore by the strong maximum principle $V$ must be a positive solution. We consider the following two cases: 
Case 1. $\left\{S_{\epsilon_{n}}\right\}$ is bounded. Without loss of the generality, suppose that $\lim _{n \rightarrow \infty} S_{\epsilon_{n}}=\tilde{L}<+\infty$. In this case, $q(s), \eta_{1}(s, \xi), \eta_{2}(s, \xi)$ and $V(s, \xi)$ are all $\tilde{L}$-periodic in $s$. Since $V$ is a positive solution of (2.13), the Hopf lemma implies that $V_{\xi}(s, 0) \geqslant \sigma_{0}>0$ for all $s \in[0, \tilde{L}]$. Hence $V_{\xi}^{\epsilon_{n}}(s, 0) \geqslant \sigma_{0} / 2$ for all $s \in \mathbb{R}$ and all large $n$, and thus $\bar{p}^{\epsilon_{n}}(\tau)=\mu V_{\xi}^{\epsilon_{n}}\left(f_{\epsilon_{n}}(\tau), 0\right) \geqslant \mu \sigma_{0} / 2$ for all $\tau$ and all large $n$, contradicting the choice of $\epsilon_{n}$.

Case 2. $\left\{S_{\epsilon_{n}}\right\}$ is unbounded. Without loss of the generality, suppose that $\lim _{n \rightarrow \infty} S_{\epsilon_{n}}=+\infty$. Since

$$
\int_{0}^{S_{\epsilon}} q^{\epsilon}(s) d s=\int_{0}^{S_{\epsilon}} \bar{p}^{\epsilon}\left(f_{\epsilon}^{-1}(s)\right) d s \leqslant \int_{0}^{S_{\epsilon}} \frac{1}{\beta_{\epsilon}\left(f_{\epsilon}^{-1}(s)\right)} d s \equiv L,
$$

we deduce that

$$
\int_{0}^{\infty} q(s) d s \leqslant L .
$$

Applying $L^{p}$ estimates to (2.13) we easily see that $\left\|V\left(s_{0}+\cdot, \cdot\right)\right\|_{C^{\frac{1+v}{2}, 1+v}([0,1] \times[0,1])}$ is uniformly bounded with respect to $s_{0} \in \mathbb{R}$. It follows that $0 \leqslant q(s)=\mu V_{\xi}(s, 0)$ is uniformly continuous in $s$. Hence from $\int_{0}^{\infty} q(s) d s<+\infty$ we can conclude that $q(s) \rightarrow 0$ as $s \rightarrow+\infty$.

Recall that $\underline{U}$ is the unique positive solution of (2.11). Let $s_{0}>0$ be chosen such that $q(s)<\delta$ for $s \geqslant s_{0}$. Then choose $\sigma>0$ such that $\sigma \underline{U}(\xi) \leqslant V\left(s_{0}, \xi\right)$ for $\xi \in[0,2 l]$. Since

$$
q(s) \underline{U}_{\xi}(\xi) \leqslant \delta(\xi) \underline{U}_{\xi}(\xi) \quad \forall \xi \in[0,2 l],
$$

we find that $\tilde{U}=\sigma \underline{U}$ satisfies

$$
\tilde{U}_{s}+q(s) \tilde{U}_{\xi}-d \tilde{U}_{\xi \xi} \leqslant \tilde{U}\left[\eta_{1}(s, \xi)-\eta_{2}(s, \xi) \tilde{U}\right] \quad \forall(s, \xi) \in \mathbb{R} \times(0, \infty) .
$$

Clearly $\tilde{U}(0)=0=V(s, 0), \tilde{U}(2 l)=0<V(s, 2 l)$ and $\tilde{U}(\xi) \leqslant V\left(s_{0}, \xi\right)$ for all $\xi \in(0,2 l)$. Hence we can apply the comparison principle to conclude that $V(s, \xi) \geqslant \tilde{U}(\xi)$ for all $s \geqslant s_{0}$ and $\xi \in[0,2 l]$. It follows that

$$
V_{\xi}(s, 0) \geqslant \tilde{U}_{\xi}(0)>0 \quad \forall s \geqslant s_{0} .
$$

On the other hand,

$$
V_{\xi}(s, 0)=\mu^{-1} q(s) \rightarrow 0 \quad \text { as } s \rightarrow \infty .
$$

This contradiction completes our proof.

\section{Uniqueness of the pulsating semi-wave and other basic properties}

If $(u(t, x), h(t))$ is a pulsating semi-wave to (1.3), then $(U(\tau, \xi), p(\tau))$ given by

$$
U(\tau, \xi)=u\left(h^{-1}(\tau), \tau-\xi\right), \quad p(\tau)=-\mu u_{x}\left(h^{-1}(\tau), \tau\right)
$$

solves (2.1) and $p(\tau)=\mu U_{\xi}(\tau, 0) \in C_{L}^{\nu}(\mathbb{R})$ is positive. So

$$
h^{\prime}(t)=p(h(t)) \geqslant \min _{\tau \in \mathbb{R}} p(\tau)>0, \quad \text { and } \quad \lim _{t \rightarrow \pm \infty} h(t)= \pm \infty .
$$

Hence, due to the $L$-periodicity of $p(\tau)$, we have

$$
\lim _{t \rightarrow \infty} \frac{h(t)}{t}=L\left(\int_{0}^{L} \frac{d s}{p(s)}\right)^{-1} .
$$

Recalling that $h^{\prime}(t)$ is $T$-periodic with $T=\int_{0}^{L} \frac{d s}{p(s)}$, we obtain

$$
\lim _{t \rightarrow \infty} h(t) / t=L / T \text {. }
$$




\subsection{Uniqueness}

To prove the uniqueness of the pulsating semi-wave, we let $\left(u^{1}, h^{1}\right)$ and $\left(u^{2}, h^{2}\right)$ be pulsating semi-waves of (1.3), and $\left(U^{1}, p^{1}\right),\left(U^{2}, p^{2}\right)$ be the corresponding pairs defined above. We have

$$
\lim _{t \rightarrow \infty} \frac{h^{i}(t)}{t}=c^{i}:=L\left(\int_{0}^{L} \frac{d s}{p^{i}(s)}\right)^{-1}, \quad i=1,2 .
$$

We will show below that $c^{1} \neq c^{2}$ leads to a contradiction, and $c^{1}=c^{2}$ implies $\left(u^{1}(t, x), h^{1}(t)\right) \equiv\left(u^{2}\left(t+t_{1}, x\right), h^{2}(t+\right.$ $\left.t_{1}\right)$ ) for some $t_{1} \in \mathbb{R}$. Such a strategy has been successfully used in [9] for the time-periodic case. Here we show that with adequate modifications, the strategy also works for the space-periodic case.

Theorem 3.1. Let $\left(u^{1}, h^{1}\right)$ and $\left(u^{2}, h^{2}\right)$ be two pulsating semi-waves of $(1.3)$. Then $\left(u^{1}(t, x), h^{1}(t)\right) \equiv\left(u^{2}(t+\right.$ $\left.\left.t_{1}, x\right), h^{2}\left(t+t_{1}\right)\right)$ for some $t_{1} \in \mathbb{R}$.

Proof. After suitable translations in $t$, we may assume that $h^{1}(0)=h^{2}(0)=0$. We now define $\left(U^{i}, p^{i}\right)(i=1,2)$ as above. Then

$$
t=\int_{0}^{h^{i}(t)} \frac{d s}{p^{i}(s)}, \quad i=1,2,
$$

and

$$
\lim _{t \rightarrow \infty} \frac{h^{i}(t)}{t}=c^{i}:=L / T_{i}, \quad T_{i}:=\int_{0}^{L} \frac{d s}{p^{i}(s)}, i=1,2 .
$$

We will show that $c^{1}=c^{2}$ implies $\left(u^{1}, h^{1}\right) \equiv\left(u^{2}, h^{2}\right)$, and $c^{1} \neq c^{2}$ leads to a contradiction. This will be done in three steps below, with the above two facts proved in Steps 1 and 2 respectively, under the assumption of a fact to be proved in Step 3.

Step 1. $c^{1}=c^{2}$ implies $\left(u^{1}, h^{1}\right) \equiv\left(u^{2}, h^{2}\right)$.

Since $c^{1}=c^{2}$, we have $T_{1}=T_{2}$. For convenience of notation we write $T_{1}=T_{2}=T$. Then $\tilde{h}(t):=h^{1}(t)-h^{2}(t)$ is a $T$-periodic function satisfying $\tilde{h}(0)=\tilde{h}(T)=0$. If $\tilde{h} \equiv 0$, then clearly $p^{1} \equiv p^{2}>0$, which implies $U^{1} \equiv U^{2}$ by applying Proposition 2.5 with $\epsilon>0$ sufficiently small (so that $p_{\epsilon}^{1}=p^{1}$ ), and hence $u^{1} \equiv u^{2}$, as we wanted. If $\tilde{h} \not \equiv 0$, we are going to derive a contradiction. In such a case, $C_{0}:=\max _{t \in \mathbb{R}} \tilde{h}(t)>0$. For each $\sigma \geq 0$ we define $\tilde{h}_{\sigma}(t):=h^{1}(t)-h^{2}(t+\sigma)$ and $C_{\sigma}=\max _{t \in \mathbb{R}} \tilde{h}_{\sigma}(t)$. It is easily seen that $C_{\sigma}$ is strictly decreasing in $\sigma$ and there exists a unique $\sigma_{0}>0$ such that $C_{\sigma_{0}}=0$. Therefore by shifting $t$ to $t+\sigma_{0}$ in $\left(u^{2}(t, x), h^{2}(t)\right)$, we may assume that, for some $t_{0} \in[0, T)$,

$$
h^{1}(t) \leqslant h^{2}(t) \quad \forall t \in \mathbb{R}, \quad h^{1}\left(t_{0}+n T\right)=h^{2}\left(t_{0}+n T\right), \quad n=0, \pm 1, \pm 2, \ldots
$$

Hence

$$
\frac{d}{d t} h^{1}\left(t_{0}+n T\right)=\frac{d}{d t} h^{2}\left(t_{0}+n T\right)
$$

To derive a contradiction, we consider the function $W(t, r):=u^{2}(t, x)-u^{1}(t, x)$ for $(t, x) \in D:=\left\{(t, x) \in \mathbb{R}^{2}: t \in \mathbb{R}\right.$, $\left.x \in\left(-\infty, h^{1}(t)\right)\right\}$. Then clearly $W(t, r) \geqslant, \not \equiv 0$ on $\partial D$ with $W\left(t_{0}, h^{1}\left(t_{0}\right)\right)=0$. We will show in Step 3 that this implies $W>0$ in $D$, and $W_{x}\left(t_{0}, h^{1}\left(t_{0}\right)\right)<0$, that is

$$
u_{x}^{1}\left(t_{0}, h^{1}\left(t_{0}\right)\right)>u_{x}^{2}\left(t_{0}, h^{2}\left(t_{0}\right)\right), \quad \text { which leads to } \frac{d}{d t} h^{1}\left(t_{0}\right)<\frac{d}{d t} h^{2}\left(t_{0}\right),
$$

a contradiction to (3.1). Thus the conclusion of Step 1 will follow if we can show that

$$
W_{x}\left(t_{0}, h^{1}\left(t_{0}\right)\right)<0 .
$$

This will be done in Step 3 below. 
Step 2. $c^{1} \neq c^{2}$ leads to a contradiction.

Without loss of generality, we assume that $c^{1}>c^{2}$. Then

$$
\lim _{t \rightarrow \pm \infty} \frac{h^{1}(t)}{t}=c^{1}>\lim _{t \rightarrow \pm \infty} \frac{h^{2}(t)}{t}=c^{2} .
$$

This implies that the curves $x=h^{1}(t)$ and $x=h^{2}(t)$ have an intersection point $\left(t_{0}, x_{0}\right)$ with a smallest $t_{0}$ value, namely

$$
h^{1}(t)<h^{2}(t) \quad \text { for } t<t_{0}, \quad h^{1}\left(t_{0}\right)=h^{2}\left(t_{0}\right)=x_{0} .
$$

It follows that

$$
\frac{d}{d t} h^{1}\left(t_{0}\right) \geqslant \frac{d}{d t} h^{2}\left(t_{0}\right)
$$

Define $W(t, r):=u^{2}(t, x)-u^{1}(t, x)$ for $(t, x) \in D_{0}:=\left\{(t, x) \in \mathbb{R}^{2}: t \in\left(-\infty, t_{0}\right], x \in\left(-\infty, h^{1}(t)\right)\right\}$. Then $W>0$ on $\partial D_{0} \backslash\left\{(t, x): t=t_{0}\right\}$ and $W\left(t_{0}, x_{0}\right)=W\left(t_{0}, h^{1}\left(t_{0}\right)\right)=0$. By Step 3 below, we have $W>0$ in $D_{0}$ and $W_{x}\left(t_{0}, x_{0}\right)<0$, which implies that

$$
\frac{d}{d t} h^{1}\left(t_{0}\right)<\frac{d}{d t} h^{2}\left(t_{0}\right)
$$

But this is in contradiction to (3.3). This completes the proof of the conclusion in Step 2, except that it remains to prove Step 3.

Step 3. Let $W$ be as in Steps 1 and 2, then $W>0$ in $D$ and $D_{0}$ respectively, and $W_{r}\left(t_{0}, h^{1}\left(t_{0}\right)\right)<0$.

We consider the case in Step 1 first. Let us recall that, by Proposition 2.3 and Remark 2.4, for $i=1,2, u^{i}(t, x) \rightarrow$ $\phi(x)$ uniformly as $h^{i}(t)-x \rightarrow+\infty$. Moreover, $u^{i}(t, x)>0$ in $D$ and $u_{x}^{i}\left(t, h^{i}(t)\right)=-U_{\xi}^{1}\left(h^{i}(t), 0\right)<0$ for all $t \in \mathbb{R}$, with $U_{\xi}^{i}\left(h^{i}(t), 0\right)$ periodic in $t$ (of period $T_{i}$ ). Hence we can find a positive constant $c_{0}>0$ such that $u^{2}(t, x) \geqslant$ $c_{0} u^{1}(t, x)$ in $D$.

Define

$$
c^{*}:=\sup \left\{c>0: u^{2}(t, x) \geqslant c u^{1}(t, x) \text { in } D\right\} .
$$

We clearly have $c^{*} \geqslant c_{0}>0$. Since $u^{i}(t, x) \rightarrow \phi(x)$ as $h^{i}(t)-x \rightarrow+\infty$, we also have $c^{*} \leqslant 1$. Thus $0<c^{*} \leqslant 1$ and

$$
W^{*}(t, x):=u^{2}(t, x)-c^{*} u^{1}(t, x) \geqslant 0 \quad \text { in } D .
$$

Using $0<c^{*} \leqslant 1$, we easily deduce from the equations for $\left(u^{i}, h^{i}\right)$ that

$$
\begin{cases}W_{t}^{*}-d W_{x x}^{*}+c(t, x) W^{*} \geqslant 0, & t \in \mathbb{R}, x<h^{1}(t), \\ W^{*}\left(t, h^{1}(t)\right) \geqslant, \not \equiv 0, & t \in \mathbb{R},\end{cases}
$$

where $c(t, x)=-a(x)+b(x)\left[u^{2}(t, x)+c^{*} u^{1}(t, x)\right]$. Thus we can apply the strong maximum principle to (3.4) to conclude that the nonnegative function $W^{*}$ is positive in $D$. Since $W^{*}\left(t_{0}, h^{1}\left(t_{0}\right)\right)=W\left(t_{0}, h^{1}\left(t_{0}\right)\right)=0$, the Hopf lemma infers that $W_{x}^{*}\left(t_{0}, h^{1}\left(t_{0}\right)\right)<0$. If we can show that $c^{*}=1$, then $W^{*}=W$ and the required fact is proved.

We use an indirect argument to show that $c^{*}=1$. Suppose by way of contradiction that $0<c^{*}<1$. Then by the definition of $c^{*}$, for any sequence of positive numbers $\epsilon_{n} \rightarrow 0$, there exists $\left(t_{n}, x_{n}\right) \in D$ such that

$$
u^{2}\left(t_{n}, x_{n}\right) \leqslant\left(c^{*}+\epsilon_{n}\right) u^{1}\left(t_{n}, x_{n}\right) \quad \forall n \geqslant 1 .
$$

We may write $t_{n}=m_{n} T+\tilde{t}_{n}$ with $m_{n}$ an integer and $\tilde{t}_{n} \in[0, T)$. Then $h^{i}\left(t_{n}\right)=h^{i}\left(\tilde{t}_{n}\right)+m_{n} L$ and $\tilde{h}\left(t_{n}\right)=\tilde{h}\left(\tilde{t}_{n}\right)$. By passing to a subsequence, we may assume that $\tilde{t}_{n} \rightarrow \tilde{t} \in[0, T]$. Then $\tilde{h}\left(t_{n}\right)=\tilde{h}\left(\tilde{t}_{n}\right) \rightarrow \tilde{h}(\tilde{t})$ as $n \rightarrow \infty$. We claim that $h^{1}\left(t_{n}\right)-x_{n}$ has an upper bound independent of $n$. Otherwise by passing to a subsequence we may assume that $h^{1}\left(t_{n}\right)-x_{n} \rightarrow \infty$ as $n \rightarrow \infty$. Then

$$
h^{2}\left(t_{n}\right)-x_{n} \geqslant h^{1}\left(t_{n}\right)-x_{n} \rightarrow \infty
$$

and hence, for $i=1,2$,

$$
u^{i}\left(t_{n}, x_{n}\right)-\phi\left(x_{n}\right) \rightarrow 0 \quad \text { as } n \rightarrow \infty .
$$


It follows from (3.5) that

$$
\limsup _{n \rightarrow \infty}\left(1-c^{*}\right) \phi\left(x_{n}\right) \leqslant 0 .
$$

On the other hand, there exists $\eta_{0}>0$ such that $\phi(x) \geqslant \eta_{0}$ for all $x \in \mathbb{R}$. Hence

$$
\limsup _{n \rightarrow \infty}\left(1-c^{*}\right) \phi\left(x_{n}\right) \geqslant\left(1-c^{*}\right) \eta_{0}>0 .
$$

This contradiction proves our claim. Thus by passing to a subsequence we may assume that $h^{1}\left(t_{n}\right)-x_{n} \rightarrow \tilde{r} \in[0, \infty)$. Then

$$
h^{2}\left(t_{n}\right)-x_{n}=h^{1}\left(t_{n}\right)-x_{n}-\tilde{h}\left(\tilde{t}_{n}\right) \rightarrow \tilde{r}-\tilde{h}(\tilde{t})
$$

and making use of (3.5), the relationship $u^{i}(t, x)=U^{i}\left(h^{i}(t), h^{i}(t)-x\right)$ and

$$
U^{i}\left(h^{i}\left(t_{n}\right), h^{i}\left(t_{n}\right)-x_{n}\right)=U^{i}\left(h^{i}\left(\tilde{t}_{n}\right), h^{i}\left(t_{n}\right)-x_{n}\right),
$$

we deduce

$$
U^{2}\left(h^{2}(\tilde{t}), \tilde{r}-\tilde{h}(\tilde{t})\right) \leqslant c^{*} U^{1}\left(h^{1}(\tilde{t}), \tilde{r}\right),
$$

that is,

$$
W^{*}\left(\tilde{t}, h^{1}(\tilde{t})-\tilde{r}\right) \leqslant 0 .
$$

Since $W^{*}>0$ in $D$, we necessarily have $\tilde{r}=0, W^{*}\left(\tilde{t}, h^{1}(\tilde{t})\right)=0$ and $W_{x}^{*}\left(\tilde{t}, h^{1}(\tilde{t})\right)<0$. By continuity we can find positive constants $\epsilon_{0}$ and $\delta_{0}$ such that

$$
W_{x}^{*}\left(t, h^{1}(t)\right)<-2 \delta_{0} \quad \forall t \in\left[\tilde{t}-\epsilon_{0}, \tilde{t}+\epsilon_{0}\right] .
$$

This implies that

$$
W^{*}\left(t_{n}, x_{n}\right)=W^{*}\left(\tilde{t}_{n}, x_{n}-m_{n} L\right) \geqslant \delta_{0}\left[h^{1}\left(\tilde{t}_{n}\right)-x_{n}+m_{n} L\right] \text { for all large } n,
$$

with

$$
x_{n}-m_{n} L=h^{1}\left(\tilde{t}_{n}\right)+x_{n}-h^{1}\left(t_{n}\right) \rightarrow h^{1}(\tilde{t})+\tilde{r}=h^{1}(\tilde{t}) \quad \text { as } n \rightarrow \infty,
$$

due to $\tilde{r}=0$ and $\tilde{t}_{n} \rightarrow \tilde{t}$. On the other hand, from $u^{1}\left(t, h^{1}(t)\right)=0$ and $u_{x}^{1}\left(t, h^{1}(t)\right)=-\left(U^{1}\right)_{x}\left(h^{1}(t), 0\right)<0$ we find that

$$
u^{1}\left(t_{n}, x_{n}\right)=u^{1}\left(\tilde{t}_{n}, x_{n}-m_{n} L\right) \leqslant M_{0}\left[h^{1}\left(\tilde{t}_{n}\right)-x_{n}+m_{n} L\right] \text { for all large } n,
$$

where $M_{0}=2 \max _{t \in[0, T]} U_{x}^{1}\left(h^{1}(t), 0\right)$. Thus for all large $n$, by (3.6), we have

$$
u^{2}\left(t_{n}, x_{n}\right) \geqslant c^{*} u^{1}\left(t_{n}, x_{n}\right)+\delta_{0}\left[h^{1}\left(\tilde{t}_{n}\right)-x_{n}+m_{n} L\right] \geqslant\left(c^{*}+\frac{\delta_{0}}{M_{0}}\right) u^{1}\left(t_{n}, x_{n}\right) .
$$

But this is in contradiction to (3.5). This proves $c^{*}=1$ and thus $W>0$ in $D$ and $W_{r}\left(t_{0}, h^{1}\left(t_{0}\right)\right)<0$, as required in Step 1.

The proof of the conclusion required in Step 2 follows a similar consideration. This time we define

$$
c^{*}:=\sup \left\{c>0: u^{2}(t, x) \geqslant c u^{1}(t, x) \forall t \leqslant t_{0}, \forall x<h^{1}(t)\right\} .
$$

We similarly have $c^{*} \geqslant c_{0}>0$ and $c^{*} \leqslant 1$. Thus

$$
W^{*}(t, x):=u^{2}(t, x)-c^{*} u^{1}(t, x) \geqslant 0 \quad \forall t \leqslant t_{0}, \forall x \leqslant h^{1}(t) .
$$

Using $0<c^{*} \leqslant 1$ and $h^{2}(t)>h^{1}(t)$ for $t<t_{0}$, we easily deduce

$$
\begin{cases}W_{t}^{*}-d W_{x x}^{*}+c(t, x) W^{*} \geqslant 0, & t \leqslant t_{0}, x<h^{1}(t), \\ W^{*}\left(t, h^{1}(t)\right)>0, & t<t_{0},\end{cases}
$$

where $c(t, x)=-a(x)+b(x)\left[u^{2}(t, x)+c^{*} u^{1}(t, x)\right]$. Thus we can apply the strong maximum principle to (3.7) to conclude that the nonnegative function $W^{*}$ is positive in $D_{0}=\left\{(t, x): t \leqslant t_{0}, x<h^{1}(t)\right\}$. Since $W^{*}\left(t_{0}, h^{1}\left(t_{0}\right)\right)=$ $W\left(t_{0}, h^{1}\left(t_{0}\right)\right)=0$, the Hopf lemma infers that $W_{x}^{*}\left(t_{0}, h^{1}\left(t_{0}\right)\right)<0$. If we can show that $c^{*}=1$, then $W^{*}=W$ and the required fact is proved. 
Suppose by way of contradiction that $0<c^{*}<1$. Then by the definition of $c^{*}$, for any sequence of positive numbers $\epsilon_{n} \rightarrow 0$, there exists $\left(t_{n}, x_{n}\right) \in D_{0}$ such that (3.5) holds. We claim that $t_{n}$ has a lower bound that is independent of $n$. Otherwise, by passing to a subsequence we may assume that $t_{n} \rightarrow-\infty$ as $n \rightarrow \infty$. Then from (3.2) we deduce $h^{2}\left(t_{n}\right)-x_{n} \geqslant h^{2}\left(t_{n}\right)-h^{1}\left(t_{n}\right) \rightarrow+\infty$ as $n \rightarrow \infty$. It follows that

$$
u^{2}\left(t_{n}, x_{n}\right)-\phi\left(x_{n}\right) \rightarrow 0 \text { as } n \rightarrow \infty .
$$

On the other hand we can show that

$$
u^{1}\left(t_{n}, x_{n}\right) \leqslant \phi\left(x_{n}\right) .
$$

To see this, we compare $u^{1}$ with $v(t, x)$ which is the unique solution of the Cauchy problem

$$
\begin{cases}v_{t}-d v_{x x}=v[a(x)-b(x) v], & t>0, x \in \mathbb{R}, \\ v(0, x)=M, & x \in \mathbb{R}\end{cases}
$$

where $M>0$ is chosen such that $\left\|u^{1}\right\|_{\infty}<M$. It is well known that $v(t, x) \rightarrow \phi(x)$ as $t \rightarrow \infty$. By the comparison principle, we have, for any $s \in \mathbb{R}^{1}, u^{1}(t+s, x) \leqslant v(t, x)$. Taking $s=s_{0}-t$ we obtain $u^{1}\left(s_{0}, x\right) \leqslant v(t, x)$. Letting $t \rightarrow \infty$ it results $u^{1}\left(s_{0}, x\right) \leqslant \phi(x)$, and the required inequality follows by taking $\left(s_{0}, x\right)=\left(t_{n}, x_{n}\right)$.

Thus we can use (3.5) to deduce $\limsup _{n \rightarrow \infty}\left(1-c^{*}\right) \phi\left(x_{n}\right) \leqslant 0$. This contradiction proves our claim. Hence we may assume, by passing to a subsequence, that $t_{n} \rightarrow \hat{t} \in\left(-\infty, t_{0}\right]$. We can now easily see that $x_{n}$ has a lower bound independent of $n$, for otherwise we may assume that $x_{n} \rightarrow-\infty$, which leads to $h^{i}\left(t_{n}\right)-x_{n} \rightarrow+\infty$ and hence

$$
u^{i}\left(t_{n}, x_{n}\right)-\phi\left(x_{n}\right) \rightarrow 0 \quad \text { as } n \rightarrow \infty,
$$

which again implies $\limsup _{n \rightarrow \infty}\left(1-c^{*}\right) \phi\left(x_{n}\right) \leqslant 0$. Thus we may assume that $x_{n} \rightarrow \hat{x} \in\left(-\infty, h^{1}(\hat{t})\right]$ as $n \rightarrow \infty$. Letting $n \rightarrow \infty$ in (3.5), we deduce $u^{2}(\hat{t}, \hat{x}) \leqslant c^{*} u^{1}(\hat{t}, \hat{x})$, that is $W^{*}(\hat{t}, \hat{x}) \leqslant 0$. Since $W^{*}>0$ in $D \cup\{(t, x): x=$ $\left.h^{1}(t), t<t_{0}\right\}$, we necessarily have $(\hat{t}, \hat{x})=\left(t_{0}, h^{1}\left(t_{0}\right)\right)$ and $W^{*}\left(t_{0}, h^{1}\left(t_{0}\right)\right)=0$. By the Hopf lemma we have $W_{x}^{*}\left(t_{0}, h^{1}\left(t_{0}\right)\right)<0$, and we can then derive a contradiction to (3.5) as before. The proof is now complete.

\subsection{Monotonicity in $t$}

We show that if $(u(t, x), h(t))$ is a pulsating semi-wave, then $u(t, x)$ is strictly increasing in $t$.

Theorem 3.2. Let $(u(t, x), h(t))$ be a pulsating semi-wave of (1.3). Then $u_{t}(t, x)>0$ for $x \leqslant h(t), t \in \mathbb{R}$.

This theorem is a simple consequence of the following lemma, which implies $u_{t} \geqslant 0$, but a simple application of the strong maximum principle then shows $u_{t}>0$.

Lemma 3.3. Suppose that $p \in C_{L}^{v}(\mathbb{R})$ and $p(\tau)>0$ in $\mathbb{R}$. Let $U(\tau, \xi)$ be the maximal nonnegative L-periodic in $\tau$ solution of (2.1). Then $U_{\tau}+U_{\xi} \geqslant 0$ in $\mathbb{R} \times(0, \infty)$.

Proof. Since $p(\tau)$ is periodic, there exists $\delta_{0}>0$ such that $p(\tau) \geqslant \delta_{0}$ for all $\tau \in \mathbb{R}$. Therefore (2.1) and (2.3) are the same for $\epsilon \in\left(0, \delta_{0}\right]$. We fix such an $\epsilon$ in $(2.3)$. In view of Proposition 2.5, $U(\tau, \xi)$ is either identically zero or $U(\tau, \xi)>0$ in $\mathbb{R} \times(0, \infty)$. In the former case, clearly $U_{\tau}+U_{\xi} \equiv 0$.

It remains to show that $U_{\tau}+U_{\xi} \geqslant 0$ in $\mathbb{R} \times(0, \infty)$ when $U>0$. Let $u(t, x)=U(h(t), h(t)-x)$ with $h(t)$ determined by

$$
h^{\prime}(t)=p(h(t)), \quad h(0)=0 .
$$

Then $u$ is a positive solution to

$$
\begin{cases}u_{t}-d u_{x x}=u[a(x)-b(x) u], & t \in \mathbb{R}, x<h(t), \\ u(t, h(t))=0, & t \in \mathbb{R} .\end{cases}
$$

Since $u_{t}=h^{\prime}(t)\left(U_{\tau}+U_{\xi}\right)$, it suffices to show that $u_{t} \geqslant 0$, or equivalently, $u(t+\sigma, x) \geqslant u(t, x)$ for any $\sigma>0$. Fix $\sigma>0$ and denote $u^{\sigma}(t, x)=u(t+\sigma, x)$. Clearly $u^{\sigma}$ satisfies (3.8) except that $h(t)$ there should be replaced by $h(t+\sigma)$. 
We first prove that there exists $M>0$ such that $M u^{\sigma}(t, x) \geqslant u(t, x)$ for $x \leqslant h(t)$ and $t \in \mathbb{R}$. From Proposition 2.5, we have

$$
\lim _{\xi \rightarrow+\infty}\left[U(\tau, \xi)-\bar{U}^{p, \epsilon}(\tau, \xi)\right]=0 \quad \text { uniformly in } \tau \in \mathbb{R} .
$$

By Remark 2.4 we have $\bar{U}^{p, \epsilon}(h(t), h(t)-x) \equiv \phi(x)$, where $\phi$ is the unique positive periodic solution of

$$
-d \phi_{x x}=\phi[a(x)-b(x) \phi], \quad x \in \mathbb{R} .
$$

It follows that

$$
\lim _{h(t)-x \rightarrow+\infty}[u(t, x)-\phi(x)]=0 \quad \text { uniformly. }
$$

Similarly

$$
\lim _{h(t)-x \rightarrow+\infty}\left[u^{\sigma}(t, x)-\phi(x)\right]=0 \quad \text { uniformly. }
$$

Therefore, for any given $M_{0}>1$, there exists $R=R_{M_{0}}>0$ such that $h(t)-x \geqslant R$ and $M \geqslant M_{0}$ imply

$$
M u^{\sigma}(t, x)-u(t, x) \geqslant \frac{M_{0}-1}{2} \phi(x)>0 .
$$

For $h(t)-x \in[0, R]$, we have

$$
u(t, x)=U(h(t), h(t)-x) \leqslant \sigma_{1}:=\max _{(\tau, \xi) \in[0, L] \times[0, R]} U(\tau, \xi)<+\infty,
$$

and

$$
u^{\sigma}(t, x)=U(h(t+\sigma), h(t+\sigma)-x) \geqslant \sigma_{2}:=\min _{(\tau, \xi) \in[0, L] \times\left[\xi_{0}, \xi^{0}\right]} U(\tau, \xi)>0,
$$

where $\xi_{0}=\inf _{t \in \mathbb{R}}[h(t+\sigma)-h(t)]>0, \xi^{0}=\sup _{t \in \mathbb{R}}[h(t+\sigma)-h(t)]+R<+\infty$. Therefore, if $M \geqslant M_{0}$ satisfies $M \sigma_{2} \geqslant \sigma_{1}$, then

$$
M u^{\sigma}(t, x) \geqslant u(t, x) \quad \text { for } x \leqslant h(t), \quad t \in \mathbb{R} .
$$

Define

$$
M_{*}:=\inf \left\{M>0: M u^{\sigma}(t, x) \geqslant u(t, x) \text { for } x \leqslant h(t), t \in \mathbb{R}\right\} .
$$

To complete the proof of the theorem, it suffices to show that $M_{*} \leqslant 1$.

In the following, we assume $M_{*}>1$ and deduce a contradiction. By definition, we have

$$
M_{*} u^{\sigma} u(t, x) \geqslant u(t, x) \quad \forall x \leqslant h(t), t \in \mathbb{R},
$$

and there exists $\left(t_{n}, x_{n}\right)$ such that

$$
x_{n} \leqslant h\left(t_{n}\right), \quad\left(M_{*}-\epsilon_{n}\right) u^{\sigma}\left(t_{n}, x_{n}\right) \leqslant u\left(t_{n}, x_{n}\right), \quad n=1,2, \ldots,
$$

where $\left\{\epsilon_{n}\right\}$ is a decreasing sequence converging to 0 as $n \rightarrow \infty$. We may assume that $M^{*}-\epsilon_{1}>1$.

Take $M_{0}=M_{*}-\epsilon_{1}$ and $M=M_{*}-\epsilon_{n}$ in (3.9), we obtain

$$
\left(M_{*}-\epsilon_{n}\right) u^{\sigma}(t, x)>u(t, x) \text { for } h(t)-x \geqslant R_{0}:=R_{M_{0}}, t \in \mathbb{R} .
$$

It follows that

$$
\xi_{n}:=h\left(t_{n}\right)-x_{n} \in\left(0, R_{0}\right), \quad n=1,2, \ldots
$$

Write

$$
h\left(t_{n}\right)=k_{n} L+\tau_{n}, \quad \text { with } k_{n} \text { an integer and } \tau_{n} \in[0, L) .
$$

We may assume that

$$
\left(\tau_{n}, \xi_{n}\right) \rightarrow\left(\tau_{\infty}, \xi_{\infty}\right) \in[0, L] \times\left[0, R_{0}\right]
$$




\section{Define}

$$
u^{n}(t, x)=u\left(t_{n}+t, x_{n}+x\right) \quad \text { for } t \in \mathbb{R}, x_{n}+x<h\left(t_{n}+t\right) .
$$

Clearly

$$
h\left(t_{n}+t\right)-x_{n}=\xi_{n}+\tilde{h}_{n}(t)
$$

with

$$
\tilde{h}_{n}(t)=h\left(t_{n}+t\right)-h\left(t_{n}\right)=\int_{0}^{t} p\left(h\left(t_{n}+s\right)\right) d s=\int_{0}^{t} p\left(\tilde{h}_{n}(s)+\tau_{n}\right) d s .
$$

Moreover, $u^{n}$ satisfies

$$
u_{t}^{n}-d u_{x x}^{n}=u^{n}\left[a\left(x_{n}+x\right)-b\left(x_{n}+x\right) u^{n}\right] \text { for } x \leqslant \xi_{n}+\tilde{h}_{n}(t)=: h_{n}(t),
$$

and

$$
u^{n}\left(t, h_{n}(t)\right) \equiv 0,
$$

with

$$
\begin{aligned}
& a\left(x_{n}+x\right)=a\left(h\left(t_{n}\right)-\xi_{n}+x\right)=a\left(\tau_{n}-\xi_{n}+x\right)=: a_{n}(x), \\
& b\left(x_{n}-x\right)=b\left(\tau_{n}-\xi_{n}+x\right)=: b_{n}(x) .
\end{aligned}
$$

Clearly

$$
a_{n}(x) \rightarrow a_{\infty}(x):=a\left(\tau_{\infty}-\xi_{\infty}+x\right), \quad b_{n}(x) \rightarrow b_{\infty}(x):=b\left(\tau_{\infty}-\xi_{\infty}+x\right)
$$

in $C_{L}^{\delta}(\mathbb{R})$. Since $\tilde{h}_{n}(\cdot)$ is uniformly continuous, by passing to a subsequence we may assume that $\tilde{h}_{n}(\cdot) \rightarrow \hat{h}(\cdot)$ in $C_{l o c}^{0}(\mathbb{R})$. It follows that

$$
\tilde{h}_{n}(t) \rightarrow h_{\infty}(t):=\int_{0}^{t} p\left(\hat{h}(s)+\tau_{\infty}\right) d s \quad \text { in } C_{l o c}^{1}(\mathbb{R}) .
$$

We may now apply standard parabolic $L^{p}$ estimates to the equation satisfied by $u^{n}$ to conclude that, subject to passing to a subsequence, $u^{n} \rightarrow u^{\infty}$ locally uniformly in $\left\{(t, x): x<h_{\infty}(t), t \in \mathbb{R}\right\}$, and $u^{\infty}$ satisfies

$$
u_{t}^{\infty}-d u_{x x}^{\infty}=u^{\infty}\left[a_{\infty}(x)-b_{\infty}(x) u^{\infty}\right] \text { for } x<h_{\infty}(t), t \in \mathbb{R} .
$$

Moreover, one may straighten the boundary $x=h_{n}(t)$ in the equation of $u^{n}$ and then apply the regularity theory to the modified equation near the straightened boundary to conclude that $u^{\infty}$ is smooth up to $x=h_{\infty}(t)$, with $u^{\infty}\left(t, h_{\infty}(t)\right) \equiv 0$.

Similarly, by passing to a further subsequence, $u^{\sigma, n}(t, x):=u^{\sigma}\left(t_{n}+t, x_{n}+x\right)$ converges to some function $v^{\infty}(t, x)$ which satisfies

$$
\begin{cases}v_{t}^{\infty}-d v_{x x}^{\infty}=v^{\infty}\left[a_{\infty}(x)-b_{\infty}(x) v^{\infty}\right], & x<h_{\infty}(t+\sigma), t \in \mathbb{R}, \\ v^{\infty}\left(t, h_{\infty}(t+\sigma)\right)=0, & t \in \mathbb{R} .\end{cases}
$$

By (3.11) and (3.12), we obtain

$$
M_{*} v^{\infty}(t, x) \geqslant u^{\infty}(t, x) \text { for } x \leqslant h_{\infty}(t), t \in \mathbb{R},
$$

and

$$
M_{*} v^{\infty}(0,0)=u^{\infty}(0,0)
$$

By (3.10), we have $v^{\infty}(0,0) \geqslant \sigma_{2}>0$. Therefore $u^{\infty}$ and $v^{\infty}$ are positive solutions, and $0<h_{\infty}(0)$. Since $M_{*}>1$, $V^{\infty}:=M_{*} v^{\infty}$ satisfies

$$
V_{t}^{\infty}-d V_{x x}^{\infty} \geqslant V^{\infty}\left[a_{\infty}(x)-b_{\infty}(x) V^{\infty}\right] \text { for } x \leqslant h_{\infty}(t+\sigma), t \in \mathbb{R} .
$$


Thus $z:=V^{\infty}-u^{\infty}$ satisfies, for some bounded $c(t, x)$,

$$
z_{t}-d z_{x x} \geqslant c(t, x) z, \quad z \geqslant 0 \text { for } x<h_{\infty}(t), t \in \mathbb{R},
$$

and $z$ achieves its minimum 0 at $(0,0)$. Since $(0,0)$ is an interior point of the domain $\left\{(t, x): x<h_{\infty}(t), t \in \mathbb{R}\right\}$, by the strong maximum principle, we deduce $z \equiv 0$. But $z$ is continuous and is positive on $x=h_{\infty}(t)$ (note that $h_{\infty}$ is strictly increasing). This contradiction completes our proof.

\subsection{Continuous dependence on parameters}

In this subsection, we show that the pulsating semi-wave $(u(t, x), h(t))$, when normalized by requiring $h(0)=0$, depends continuously on $(d, \mu, a, b) \in \mathbb{R}^{2} \times C_{L}^{\nu}(\mathbb{R})^{2}$.

Theorem 3.4. Suppose that, as $n \rightarrow \infty,\left(d_{n}, \mu_{n}, a_{n}, b_{n}\right) \rightarrow\left(d_{0}, \mu_{0}, a_{0}, b_{0}\right)$ in $\mathbb{R}^{2} \times C_{L}^{v}(\mathbb{R})^{2}$, with $d_{0}, \mu_{0}>0$, $a_{0}(x), b_{0}(x)>0$. Let $\left(u^{n}, h^{n}\right)$ denote the unique normalized pulsating semi-wave of $(1.3)$, with $(d, \mu, a, b)=$ $\left(d_{n}, \mu_{n}, a_{n}, b_{n}\right), n=0,1,2, \ldots$ Then $\lim _{n \rightarrow \infty}\left[h^{n}(t)-h^{0}(t)\right]=0$ uniformly in $\mathbb{R}$, and $\lim _{n \rightarrow \infty}\left[u^{n}(t, x)-\right.$ $\left.u^{0}(t, x)\right]=0$ locally uniformly in $\left\{(t, x): x<h^{0}(t), t \in \mathbb{R}\right\}$.

Proof. Choose positive constants $A$ and $B$ such that $A \geqslant \max a_{n}, B \leqslant \min b_{n}$ for $n=0,1,2, \ldots$ Let $v(\xi)$ be the unique positive solution to

$$
-v^{\prime \prime}=v(A-B v) \quad \text { for } \xi>0, v(0)=0 .
$$

Then it is well known (see [10]) that $v^{\prime}(\xi)>0$ and $v(+\infty)=A / B$. We define $v_{n}(\xi)=v\left(\xi / \sqrt{d_{n}}\right)$. Clearly

$$
-d_{n} v_{n}^{\prime \prime}=v_{n}\left(A-B v_{n}\right) \quad \text { for } \xi>0, v_{n}(0)=0 .
$$

Let $\left(U^{n}(\tau, \xi), p^{n}(\tau)\right)$ be the corresponding pair to $\left(u^{n}(x), h^{n}(t)\right)$, as before. Then

$$
\begin{aligned}
& p^{n}(\tau)\left(U_{\tau}^{n}+\hat{U}_{\xi}\right)-d_{n} U_{\xi \xi}^{n}=U^{n}\left[a_{n}(\tau-\xi)-b_{n}(\tau-\xi) U^{n}\right], \quad(\tau, \xi) \in \mathbb{R} \times[0, \infty), \\
& U^{n}(\tau, 0)=0, \quad p^{n}(\tau)=\mu_{n} U_{\xi}^{n}(\tau, 0), \quad \tau \in \mathbb{R},
\end{aligned}
$$

and much as at the beginning of the proof of Proposition 2.5, we can use a comparison argument to conclude that $U^{n}(\tau, \xi) \leqslant v_{n}(\xi)$ for $\tau \in \mathbb{R}$ and $\xi>0$. It follows that $U_{\xi}^{n}(\tau, 0) \leqslant v_{n}^{\prime}(0)$ and hence

$$
0<p^{n}(\tau) \leqslant \mu_{n} v_{n}^{\prime}(0)=\frac{\mu_{n}}{\sqrt{d_{n}}} v^{\prime}(0), \quad n=0,1,2, \ldots
$$

Thus $\left\{p^{n}\right\}$ is bounded in $C_{L}^{0}(\mathbb{R})$. Checking the proof of Proposition 2.9 we find that there exists $\epsilon>0$ independent of $n$ such that $p^{n}(\tau) \geqslant \epsilon$ for all $\tau \in \mathbb{R}$ and $n=0,1,2, \ldots$. Therefore, in view of $0<U^{n} \leqslant v_{n} \leqslant A / B$, we may apply standard $L^{p}$ theory for parabolic equations to conclude that $\left\{U^{n}\right\}$ is a bounded set in $W_{q}^{1,2}([0, L] \times[0, M])$ $(\forall q>1, \forall M>0)$. By Sobolev embedding we see that $\left\{U^{n}\right\}$ is a bounded set in $C^{\frac{1+v_{0}}{2}}, 1+v_{0}([0, L] \times[0, M])$ with $\nu_{0} \in(\nu, 1)$. Therefore by passing to a subsequence we may assume that $p^{n}(\tau)=\mu U_{\xi}^{n}(\tau, 0) \rightarrow \hat{p}(\tau)$ in $C_{L}^{v}(\mathbb{R})$. By a standard diagonal process and by passing to a further subsequence if necessary, we may assume that $U^{n} \rightarrow \hat{U}$ in $C_{l o c}^{\frac{v+1}{2}, v+1}([0, L] \times[0, \infty))$. Thus $(\hat{U}, \hat{p})$ satisfies

$$
\begin{aligned}
& \hat{p}(\tau)\left(\hat{U}_{\tau}+\hat{U}_{\xi}\right)-d_{0} \hat{U}_{\xi \xi}=\hat{U}\left[a_{0}(\tau-\xi)-b_{0}(\tau-\xi) \hat{U}\right], \quad(\tau, \xi) \in \mathbb{R} \times[0, \infty), \\
& \hat{U}(\tau, 0)=0, \quad \hat{p}(\tau)=\mu_{0} \hat{U}_{\xi}(\tau, 0), \quad \tau \in \mathbb{R} .
\end{aligned}
$$

Therefore $(\hat{u}(t, x), \hat{h}(t))$ defined by

$$
\hat{u}(t, x)=\hat{U}(\hat{h}(t), \hat{h}(t)-x), \quad t=\int_{0}^{\hat{h}(t)} \frac{1}{\hat{p}(\tau)} d \tau
$$

is a normalized pulsating semi-wave of $(1.3)$ with $(d, \mu, a, b)=\left(d_{0}, \mu_{0}, a_{0}, b_{0}\right)$. By uniqueness, necessarily $(\hat{u}, \hat{h})=$ $\left(u^{0}, h^{0}\right)$. This implies that the entire sequence $\left(u^{n}, h^{n}\right)$ converges to $\left(u^{0}, h^{0}\right)$ as stated in the theorem. 


\section{Spreading speed}

In this section we study the spreading speed determined by (1.1) when spreading occurs. We also investigate how this speed varies with $\mu$.

Let $(\tilde{u}, \tilde{h})$ be a pulsating semi-wave of $(1.3)$, which is unique up to a translation of $t$. Let the function pair $(U, p)$ be given by

$$
U(\tau, \xi)=\tilde{u}\left(\tilde{h}^{-1}(\tau), \tau-\xi\right), \quad p(\tau)=-\mu \tilde{u}_{x}\left(\tilde{h}^{-1}(\tau), \tau\right) .
$$

Then we have

$$
\tilde{h}^{\prime}(t)=p(\tilde{h}(t)) \quad \text { and } \quad \lim _{t \rightarrow \infty} \tilde{h}(t) / t=L / T \quad \text { with } T=\int_{0}^{L} \frac{d s}{p(s)} .
$$

Let us note that the first identity in the line above implies that

$$
\int_{\tilde{h}\left(t_{0}\right)}^{\tilde{h}\left(t_{0}+t\right)} \frac{d s}{p(s)}=t \quad \forall t_{0}, t \in \mathbb{R} .
$$

This implies that if $(\hat{U}, \hat{p})$ is obtained from a pulsating semi-wave which is a shift of $(\tilde{u}, \tilde{h})$ in $t$, then $\hat{p}(\tau)$ is identical to $p\left(\tau+\tau_{0}\right)$ for some $\tau_{0} \in \mathbb{R}$. Since $p(\tau)$ is $L$-periodic, we find that $\int_{0}^{L} \frac{d s}{p(s)}$ is independent of the choice of $(\tilde{u}, \tilde{h})$. Thus the quantity $L / T$ is uniquely determined.

Our first main result of this section is the following.

Theorem 4.1. Suppose that $(u, h)$ is the unique solution of $(1.1)$ and $\lim _{t \rightarrow \infty} h(t)=+\infty$. Then

$$
\lim _{t \rightarrow \infty} \frac{h(t)}{t}=\lim _{t \rightarrow \infty} \frac{\tilde{h}(t)}{t}=\frac{L}{T} .
$$

Proof. Fix $\epsilon>0$ small and for $\sigma \in\{\epsilon,-\epsilon\}$, we denote by $\left(u^{\sigma}, h^{\sigma}\right)$ the unique pulsating semi-wave of

$$
\begin{cases}u_{t}-d u_{x x}=u([a(x)+\sigma]-[b(x)-\sigma] u), & x<h(t), t \in \mathbb{R}, \\ h^{\prime}(t)=-(1-\sigma) \mu u_{x}(t, h(t)), \quad u(t, h(t))=0, & t \in \mathbb{R},\end{cases}
$$

normalized by $h^{\sigma}(0)=0$. Define

$$
\tilde{u}^{\sigma}(t, x)=u^{\sigma}\left(\frac{t}{1-\sigma}, x\right), \quad \tilde{h}^{\sigma}(t)=h^{\sigma}\left(\frac{t}{1-\sigma}\right) .
$$

Then

$$
\begin{cases}(1-\sigma) \tilde{u}_{t}^{\sigma}-d \tilde{u}_{x x}^{\sigma}=\tilde{u}^{\sigma}\left([a(x)+\sigma]-[b(x)-\sigma] \tilde{u}^{\sigma}\right), & x<\tilde{h}^{\sigma}(t), t \in \mathbb{R}, \\ \left(\tilde{h}^{\sigma}\right)^{\prime}(t)=-\mu \tilde{u}_{x}^{\sigma}\left(t, \tilde{h}^{\sigma}(t)\right), \quad \tilde{u}^{\sigma}\left(t, \tilde{h}^{\sigma}(t)\right)=0, & t \in \mathbb{R} .\end{cases}
$$

Set

$$
\begin{aligned}
& \left(\bar{u}^{\epsilon}(t, x), \bar{h}^{\epsilon}(t)\right)=\left(\tilde{u}^{\epsilon}\left(t+C_{1}, x\right), \tilde{h}^{\epsilon}\left(t+C_{1}\right)\right), \\
& \left(\underline{u}^{\epsilon}(t, x), \underline{h}^{\epsilon}(t)\right)=\left(\tilde{u}^{-\epsilon}\left(t+C_{2}, x\right), \tilde{h}^{-\epsilon}\left(t+C_{2}\right)\right) .
\end{aligned}
$$

We will show that, for all small $\epsilon>0$ and suitable $C_{1}, C_{2}, M \in \mathbb{R}$,

$$
\begin{cases}\underline{u}^{\epsilon}(t, r) \leqslant u(t, r) \leqslant \bar{u}^{\epsilon}(t, r) & \text { for } t \geqslant M, r \in\left[M, \underline{h}^{\epsilon}(t)\right], \\ \underline{h}^{\epsilon}(t) \leqslant h(t) \leqslant \bar{h}^{\epsilon}(t) & \text { for } t \geqslant M .\end{cases}
$$

If (4.2) is proved, then we have

$$
\lim _{t \rightarrow \infty} \frac{\underline{h}^{\epsilon}(t)}{t} \leqslant \liminf _{t \rightarrow \infty} \frac{h(t)}{t} \leqslant \limsup _{t \rightarrow \infty} \frac{h(t)}{t} \leqslant \lim _{t \rightarrow \infty} \frac{\bar{h}^{\epsilon}(t)}{t} .
$$


Clearly

$$
\lim _{t \rightarrow \infty} \frac{\underline{h}^{\epsilon}(t)}{t}=\lim _{t \rightarrow \infty} \frac{h^{-\epsilon}\left(\left(t+C_{2}\right) /(1+\epsilon)\right)}{t}=(1+\epsilon)^{-1} L / T_{\epsilon},
$$

and

$$
\lim _{t \rightarrow \infty} \frac{\bar{h}^{\epsilon}(t)}{t}=\lim _{t \rightarrow \infty} \frac{h^{\epsilon}\left(\left(t+C_{1}\right) /(1-\epsilon)\right)}{t}=(1-\epsilon)^{-1} L / T^{\epsilon},
$$

where $T_{\epsilon}=\int_{0}^{L} \frac{d s}{\underline{p}^{\epsilon}(s)}$, with $\underline{p}^{\epsilon}(\tau):=-\mu \underline{u}_{x}^{\epsilon}\left(\left(\underline{h}^{\epsilon}\right)^{-1}(\tau), \tau\right) ; T^{\epsilon}$ is defined analogously from $\left(\bar{u}^{\epsilon}, \bar{h}^{\epsilon}\right)$.

It follows that

$$
(1+\epsilon)^{-1} L / T_{\epsilon} \leqslant \liminf _{t \rightarrow \infty} \frac{h(t)}{t} \leqslant \limsup _{t \rightarrow \infty} \frac{h(t)}{t} \leqslant(1-\epsilon)^{-1} L / T^{\epsilon} .
$$

Letting $\epsilon \rightarrow 0$, we deduce

$$
\lim _{t \rightarrow \infty} \frac{h(t)}{t}=\frac{L}{T}
$$

as desired.

It remains to prove (4.2). This will follow from the comparison principle by showing that $\left(\bar{u}^{\epsilon}, \bar{h}^{\epsilon}\right)$ and $\left(\underline{u}^{\epsilon}, \underline{h}^{\epsilon}\right)$ are respectively upper and lower solutions to (1.1) for $t \geqslant M$. We break the argument below into several steps.

Step 1. For any large $R=R_{\epsilon}>0$ satisfying

$$
\alpha(r) \in\left[a(r)-\frac{\epsilon}{2}, a(r)+\frac{\epsilon}{2}\right], \quad \beta(r) \in\left[b(r)-\frac{\epsilon}{2}, b(r)+\frac{\epsilon}{2}\right] \quad \forall r \geqslant R,
$$

there exists $\tau_{R}>0$ such that

$$
\begin{array}{ll}
\underline{u}_{t}^{\epsilon}-d \Delta \underline{u}^{\epsilon} \leqslant \underline{u}^{\epsilon}\left[\alpha(r)-\beta(r) \underline{u}^{\epsilon}\right], & r \in\left[\tau_{R}, \underline{h}^{\epsilon}(t)\right], \\
\bar{u}_{t}^{\epsilon}-d \Delta \bar{u}^{\epsilon} \geqslant \bar{u}^{\epsilon}\left[\alpha(r)-\beta(r) \bar{u}^{\epsilon}\right], & r \in\left[\tau_{R}, \bar{h}^{\epsilon}(t)\right],
\end{array}
$$

with the inequalities satisfied for all $t$ such that $\underline{h}^{\epsilon}(t)>\tau_{R}$ and $\bar{h}^{\epsilon}(t)>\tau_{R}$, respectively.

To prove (4.3), we note that

$$
\begin{aligned}
& \underline{u}^{\epsilon}\left\{[a(r)-\epsilon]-[b(r)+\epsilon] \underline{u}^{\epsilon}\right\}+\frac{\epsilon}{2} \underline{u}^{\epsilon} \\
& \quad \leqslant \underline{u}^{\epsilon}\left[\alpha(r)-\beta(r) \underline{u}^{\epsilon}\right] \quad \text { for } r \in\left[R, \underline{h}^{\epsilon}(t)\right] .
\end{aligned}
$$

Therefore, in view of the equation satisfied by $\underline{u}^{\epsilon}$, (4.3) will follow if we have

$$
\begin{aligned}
\underline{u}_{t}^{\epsilon} & -d\left(\underline{u}_{r r}^{\epsilon}+\frac{N-1}{r} \underline{u}_{r}^{\epsilon}\right) \\
& \leqslant(1+\epsilon) \underline{u}_{t}^{\epsilon}-d \underline{u}_{r r}^{\epsilon}+\frac{\epsilon}{2} \underline{u}^{\epsilon} \quad \text { for } r \in\left[\tau_{R}, \underline{h}^{\epsilon}(t)\right],
\end{aligned}
$$

or equivalently,

$$
-d \frac{N-1}{r} \underline{u}_{r}^{\epsilon} \leqslant \epsilon \underline{u}_{t}^{\epsilon}+\frac{\epsilon}{2} \underline{u}^{\epsilon} \quad \text { for } r \in\left[\tau_{R}, \underline{h}^{\epsilon}(t)\right] .
$$

From the identity $\underline{u}^{\epsilon}\left(t, \underline{h}^{\epsilon}(t)\right) \equiv 0$, we deduce

$$
\underline{u}_{t}^{\epsilon}(t, r)+\left(\underline{h}^{\epsilon}\right)^{\prime}(t) \underline{u}_{r}^{\epsilon}(t, r)=0 \quad \text { for } r=\underline{h}^{\epsilon}(t) .
$$

Therefore

$$
-\underline{u}_{r}^{\epsilon}(t, r)=\left[\left(\underline{h}^{\epsilon}\right)^{\prime}(t)\right]^{-1} \underline{u}_{t}^{\epsilon}(t, r)>0 \quad \text { for } r=\underline{h}^{\epsilon}(t) .
$$

Moreover, the functions $\underline{u}^{\epsilon}\left(t, \underline{h}^{\epsilon}(t)\right), \underline{u}_{t}^{\epsilon}\left(t, \underline{h}^{\epsilon}(t)\right)$ and $\underline{u}_{r}^{\epsilon}\left(t, \underline{h}^{\epsilon}(t)\right)$ are periodic in $t$ (recalling the transform $u(t, x)=$ $U(h(t), h(t)-x))$. Hence, from the uniform continuity of $\underline{u}^{\epsilon}, \underline{u}_{t}^{\epsilon}$ and $\underline{u}_{r}^{\epsilon}$ on $(t, \xi)=\left(t, \underline{h}^{\epsilon}(t)-r\right)$, we can find $\delta=\delta_{\epsilon}>0$ such that

$$
-\underline{u}_{r}^{\epsilon}(t, r) \leqslant \sigma_{0} \underline{u}_{t}^{\epsilon}(t, r) \quad \text { for } r \in\left[\underline{h}^{\epsilon}(t)-\delta, \underline{h}^{\epsilon}(t)\right], t \in \mathbb{R},
$$


where $\sigma_{0}=2 \max _{t \in \mathbb{R}}\left[\left(\underline{h}^{\epsilon}\right)^{\prime}(t)\right]^{-1}$. It follows that (4.5) holds in the range $r \in\left[\underline{h}^{\epsilon}(t)-\delta, \underline{h}^{\epsilon}(t)\right]$ provided that $\underline{h}^{\epsilon}(t)-$ $\delta>R_{1}$, where $R_{1}:=d(N-1) \sigma_{0} / \epsilon$.

For $r \in\left(-\infty, \underline{h}^{\epsilon}(t)-\delta\right]$, we have $\underline{u}^{\epsilon} \geqslant \sigma_{1}>0$ and $\left|\underline{u}_{r}^{\epsilon}\right| \leqslant \sigma_{2}<+\infty$. Thus (4.5) holds for $r \in\left[\tau_{R}, \underline{h}^{\epsilon}(t)\right]$ with

$$
\tau_{R}:=\max \left\{\frac{2 d(N-1) \sigma_{2}}{\epsilon \sigma_{1}}, R_{1}, R\right\} .
$$

The proof of (4.4) is similar and we omit the details.

Step 2. There exist $R_{0} \geqslant \tau_{R}$ and $T_{1}>0$ such that,

$$
\bar{h}^{\epsilon}\left(T_{1}\right)>h\left(T_{1}\right), \quad \underline{h}^{\epsilon}\left(T_{1}\right)<R_{0}<h\left(T_{1}\right)
$$

and

$$
\bar{u}^{\epsilon}\left(t, R_{0}\right) \geqslant u\left(t, R_{0}\right), \quad \underline{u}^{\epsilon}\left(t, R_{0}\right) \leqslant u\left(t, R_{0}\right) \quad \text { for } t \geqslant T_{1} .
$$

For $\sigma \in\{\epsilon, 0,-\epsilon\}$, let $\phi^{\sigma}(x)$ be the unique positive solution of

$$
-d \phi_{x x}=\phi\{[a(x)+\sigma]-[b(x)-\sigma] \phi\}, \quad x \in \mathbb{R} .
$$

Then $\phi^{\sigma}$ is $L$-periodic and $\phi^{\epsilon}>\phi^{0}>\phi^{-\epsilon}$. Hence there exists $\delta=\delta_{\epsilon}>0$ such that

$$
\phi^{\epsilon}(x)-2 \delta \geqslant \phi^{0}(x) \geqslant \phi^{-\epsilon}(x)+2 \delta, \quad x \in \mathbb{R} .
$$

Let $(u(t, r), h(t))$ be the unique solution of (1.1), for which spreading occurs. By Theorem 2.4 of [7], we have

$$
\lim _{t \rightarrow \infty} u(t, r)=\hat{U}(r) \quad \text { locally uniformly for } r \in[0, \infty),
$$

where $\hat{U}$ is the unique positive solution of

$$
-d\left(U^{\prime \prime}+\frac{N-1}{r} U^{\prime}\right)=U[a(r)-b(r) U] \text { for } r \in[0, \infty) \cdot U^{\prime}(0)=0 .
$$

For any sequence $r_{n} \rightarrow \infty$, write $r_{n}=m_{n} L+\tilde{r}_{n}$ with $m_{n}$ an integer and $\tilde{r}_{n} \in[0, L)$, and define $U_{n}(r)=\hat{U}\left(r_{n}+r\right)$. Then by passing to a subsequence one may assume that $\tilde{r}_{n} \rightarrow r_{*}$. Applying the $L^{p}$ theory to the equation of $U_{n}$, and using Sobolev embedding, one finds that by passing to a further subsequence, $U_{n} \rightarrow U_{*}$ in $C_{l o c}^{1}(\mathbb{R})$, and $U_{*}$ satisfies

$$
-d U_{*}^{\prime \prime}=U^{*}\left[a\left(r_{*}+r\right)-b\left(r_{*}+r\right) U_{*}\right] \text { in } \mathbb{R} .
$$

A simple upper and lower solution consideration shows that $U_{*}>0$. Therefore necessarily $U_{*}(r)=\phi^{0}\left(r_{*}+r\right)$. This implies that

$$
\lim _{r \rightarrow \infty}\left[\hat{U}(r)-\phi^{0}(r)\right]=0 .
$$

It follows that, with the above chosen $\delta$, there exist $R_{0} \geqslant \tau_{R}$ and $T_{0}>0$ such that

$$
u\left(t, R_{0}\right) \in\left[\phi^{0}\left(R_{0}\right)-\delta, \phi^{0}\left(R_{0}\right)+\delta\right] \text { for } t \geqslant T_{0} .
$$

On the other hand, we have

$$
\lim _{\bar{h}^{\epsilon}(t)-x \rightarrow \infty}\left[\bar{u}^{\epsilon}(t, x)-\phi^{\epsilon}(x)\right]=0, \quad \lim _{\underline{h}^{\epsilon}(t)-x \rightarrow \infty}\left[\underline{u}^{\epsilon}(t, x)-\phi^{-\epsilon}(x)\right]=0 .
$$

It follows that

$$
\lim _{t \rightarrow \infty}\left[\bar{u}^{\epsilon}\left(t, R_{0}\right)-\phi^{\epsilon}\left(R_{0}\right)\right]=0, \quad \lim _{t \rightarrow \infty}\left[\underline{u}^{\epsilon}\left(t, R_{0}\right)-\phi^{-\epsilon}\left(R_{0}\right)\right]=0 .
$$

Therefore by enlarging $T_{0}$ properly we may assume that

$$
\bar{u}^{\epsilon}\left(t, R_{0}\right) \geqslant \phi^{\epsilon}\left(R_{0}\right)-\delta, \quad \underline{u}^{\epsilon}\left(t, R_{0}\right) \leqslant \phi^{-\epsilon}\left(R_{0}\right)+\delta \quad \text { for } t \geqslant T_{0} .
$$

Hence

$$
\bar{u}^{\epsilon}\left(t, R_{0}\right) \geqslant \phi^{0}\left(R_{0}\right)+\delta \geqslant u\left(t, R_{0}\right), \quad \underline{u}^{\epsilon}\left(t, R_{0}\right) \leqslant \phi^{0}\left(R_{0}\right)-\delta \leqslant u\left(t, R_{0}\right) \quad \text { for } t \geqslant T_{0} .
$$


Recall that

$$
\bar{h}^{\epsilon}(t)=h^{\epsilon}\left(\frac{t+C_{1}}{1+\epsilon}\right), \quad \underline{h}^{\epsilon}(t)=h^{-\epsilon}\left(\frac{t+C_{2}}{1-\epsilon}\right),
$$

and in the above discussion, we only used an arbitrarily fixed pair $\left(C_{1}, C_{2}\right)$. Next we choose $C_{1}$ and $C_{2}$ to meet the required inequalities of this step. Since $\bar{u}^{\epsilon}(t, x)$ and $\underline{u}^{\epsilon}(t, x)$ are both increasing in $t$, we find that the inequalities in (4.6) remain valid when $C_{1}$ is increased and $C_{2}$ is decreased. We first choose $T_{1} \geqslant T_{0}$ such that $h\left(T_{1}\right)>R_{0}$. We may then increase $C_{1}$ and decrease $C_{2}$ properly to guarantee that

$$
\bar{h}^{\epsilon}\left(T_{1}\right)>h\left(T_{1}\right), \quad \underline{h}^{\epsilon}\left(T_{1}\right)<R_{0} .
$$

This completes the proof of Step 2.

Step 3. Completion of the proof.

Since $\underline{h}^{\epsilon}\left(T_{1}\right)<R_{0}<h\left(T_{1}\right)$, there exists $T_{2}>T_{1}$ such that

$$
\underline{h}^{\epsilon}\left(T_{2}\right)=R_{0}<h\left(T_{2}\right) .
$$

Therefore we can find $\eta>0$ sufficiently small such that

$$
R_{0}<\underline{h}^{\epsilon}\left(T_{2}+\eta\right)<h\left(T_{2}+\eta\right), \quad \underline{u}^{\epsilon}\left(T_{2}+\eta, r\right)<u\left(T_{2}+\eta, r\right) \quad \text { for } r \in\left[R_{0}, \underline{h}^{\epsilon}\left(T_{2}+\eta\right)\right] .
$$

We may now use the conclusions in Steps 1 and 2, and apply the comparison principle (see Lemma 3.3 in [7]), to conclude that

$$
h(t) \geqslant \underline{h}^{\epsilon}(t), \quad u(t, r) \geqslant \underline{u}^{\epsilon}(t, r) \quad \text { for } r \in\left[R_{0}, \underline{h}^{\epsilon}(t)\right], t \geqslant T_{2}+\eta .
$$

Let $V(t, r)$ be the unique solution of

$$
V_{t}-d \Delta V=V[\alpha(r)-\beta(r) V], \quad r \in[0, \infty), \quad V_{r}(t, 0)=0, \quad V(0, r)=\left\|u_{0}\right\|_{\infty} .
$$

Then it is well known that $\lim _{t \rightarrow \infty}\|V(t, \cdot)-\hat{U}(\cdot)\|_{L^{\infty}([0, \infty))}=0$. By the comparison principle we have $u(t, r) \leqslant$ $V(t, r)$ for $r \in[0, h(t)], t>0$. It follows that $\limsup _{t \rightarrow \infty} u(t, r) \leqslant \hat{U}(r)$ uniformly for $r \in[0, \infty)$. Therefore, if $R_{0}$ and $T_{0}$ are large enough in Step 2, we have

$$
u(t, r) \leqslant \phi^{0}(r)+\delta \leqslant \phi^{\epsilon}(r)-\delta \text { for } r \in\left[R_{0}, h(t)\right], t \geqslant T_{0},
$$

where $\delta>0$ is as in Step 2. If $C_{1}$ is sufficiently large, we can guarantee that

$$
\bar{u}^{\epsilon}\left(T_{1}, r\right) \geqslant \phi^{\epsilon}(r)-\delta \geqslant u\left(T_{1}, r\right) \text { for } r \in\left[R_{0}, h\left(T_{1}\right)\right] .
$$

Therefore we can apply the comparison principle (see Lemma 3.2 of [7]) to conclude that

$$
\bar{h}^{\epsilon}(t) \geqslant h(t), \quad \bar{u}^{\epsilon}(t, r) \geqslant u(t, r) \quad \text { for } r \in\left[R_{0}, h(t)\right], t \geqslant T_{1} .
$$

The proof is now complete.

Next we investigate the dependence of the spreading speed on the parameter $\mu$. To stress this dependence, we will write $T=T(\mu)$ and $C(\mu)=L / T(\mu)$. We will prove the following result.

Theorem 4.2. $C(\mu)$ is strictly increasing in $\mu$, and $\lim _{\mu \rightarrow \infty} C(\mu)$ exists and equals $c_{*}$, which is the minimal speed of the pulsating fronts for (1.4) obtained in [5].

The proof of this theorem is broken into several lemmas.

Lemma 4.3. Suppose that $0<\mu_{1}<\mu_{2}$. Then $C\left(\mu_{1}\right)<C\left(\mu_{2}\right)$.

Proof. Let $\left(u_{1}, h_{1}\right)$ and $\left(u_{2}, h_{2}\right)$ be the unique solutions of (1.1) with $\mu=\mu_{1}$ and $\mu_{2}$, respectively. Suppose that $h_{1}(\infty)=\infty$. By Theorem 3.5 of [8], we see that $h_{1}(t) \leqslant h_{2}(t)$ for $t>0$. It follows from Theorem 4.1 that $C\left(\mu_{1}\right) \leqslant$ $C\left(\mu_{2}\right)$. 
It remains to show $C\left(\mu_{1}\right) \neq C\left(\mu_{2}\right)$. Let $\left(u^{1}, h^{1}\right)$ and $\left(u^{2}, h^{2}\right)$ be pulsating semi-waves corresponding to $\left(u_{1}, h_{1}\right)$ and $\left(u_{2}, h_{2}\right)$ respectively. By Theorem 4.1,

$$
\lim _{t \rightarrow \infty} \frac{h^{i}(t)}{t}=C\left(\mu_{i}\right), \quad i=1,2 .
$$

Arguing indirectly we suppose that $C\left(\mu_{1}\right)=C\left(\mu_{2}\right)$; we are going to deduce a contradiction. We follow the argument in the proof of Theorem 3.1. As in Step 1 there, our assumption implies that $\tilde{h}(t):=h^{1}(t)-h^{2}(t)$ is a $T$-periodic function satisfying $\tilde{h}(0)=\tilde{h}(T)=0$. If $\tilde{h} \equiv 0$, then $\tilde{u}^{1} \equiv \tilde{u}^{2}$ and

$$
\frac{d}{d t} h^{1}(t)=-\mu_{1} u_{x}^{1}\left(t, h^{1}(t)\right) \equiv-\mu_{1} u_{x}^{2}\left(t, h^{2}(t)\right)=\frac{\mu_{1}}{\mu_{2}} \frac{d}{d t} h^{2}(t)<\frac{d}{d t} h^{2}(t),
$$

which is a contradiction.

If $\tilde{h} \not \equiv 0$, then as in Step 1 of the proof of Theorem 3.1, we can shift $t$ in $h^{2}(t)$ so that, after the shift,

$$
h^{1}(t) \leqslant h^{2}(t) \quad \forall t \in \mathbb{R}, \quad h^{1}\left(t_{0}+n T\right)=h^{2}\left(t_{0}+n T\right), \quad n=0, \pm 1, \pm 2, \ldots
$$

It follows that

$$
\frac{d}{d t} h^{1}\left(t_{0}+n T\right)=\frac{d}{d t} h^{2}\left(t_{0}+n T\right) .
$$

On the other hand, if we define

$$
W(t, x)=u^{2}(t, x)-u^{1}(t, x) \quad \text { for }(t, x) \in D:=\left\{(t, x): t \in \mathbb{R}, x<h^{1}(t)\right\},
$$

then the same arguments as in the proof of Theorem 3.1 yield

$$
W(t, x)>0 \quad \text { in } D \quad \text { and } \quad W_{x}\left(t_{0}, h^{1}\left(t_{0}\right)\right)<0 .
$$

It follows that

$$
u_{x}^{1}\left(t_{0}, h^{1}\left(t_{0}\right)\right)>u_{x}^{2}\left(t_{0}, h^{2}\left(t_{0}\right)\right),
$$

or equivalently

$$
-\mu_{1}^{-1} \frac{d}{d t} h^{1}\left(t_{0}\right)>-\mu_{2}^{-1} \frac{d}{d t} h^{2}\left(t_{0}\right),
$$

which implies, due to $\mu_{1}<\mu_{2}$,

$$
\frac{d}{d t} h^{1}\left(t_{0}\right)<\frac{d}{d t} h^{2}\left(t_{0}\right)
$$

Again we reach a contradiction.

Since every possibility leads to a contradiction, we conclude that $C\left(\mu_{1}\right) \neq C\left(\mu_{2}\right)$.

Lemma 4.4. Suppose that $0<\mu_{1}<\mu_{2}$, and $\left(u^{1}, h^{1}\right),\left(u^{2}, h^{2}\right)$ are the unique pulsating semi-waves of (1.3) with $\mu=\mu_{1}, \mu_{2}$, respectively, which are normalized by $h^{1}(0)=h^{2}(0)=0$. Then $h^{1}(t)<h^{2}(t)$ for $t>0$ and $h^{1}(t)>h^{2}(t)$ for $t<0$.

Proof. From the previous lemma we find that

$$
\lim _{t \rightarrow \infty} \frac{h^{1}(t)}{t}<\lim _{t \rightarrow \infty} \frac{h^{2}(t)}{t} .
$$

Hence $0<h^{1}(t)<h^{2}(t)$ for all large positive $t$, and $0>h^{1}(t)>h^{2}(t)$ for all large negative $t$. Therefore there exists a smallest $t_{0} \geqslant 0$ such that $h^{1}\left(t_{0}\right)=h^{2}\left(t_{0}\right)$ and $h^{1}(t)<h^{2}(t)$ for $t>t_{0}$. We show that $t_{0}=0$. Otherwise $t_{0}>0$ and due to $h^{1}(0)=h^{2}(0)=0, C_{0}:=\max _{t \in\left[0, t_{0}\right]} \tilde{h}(t) \geqslant 0$, where $\tilde{h}(t):=h^{1}(t)-h^{2}(t)$. If $C_{0}=0$ then clearly $\frac{d}{d t} h^{1}\left(t_{0}\right)=\frac{d}{d t} h^{2}\left(t_{0}\right)$. If $C_{0}>0$ then we can shift $t$ in $h^{2}(t)$ suitably to guarantee that for the shifted $h^{2}$, still denoted by $h^{2}(t)$, and some new $t_{0}>0$, one has $h^{1}(t)<h^{2}(t)$ for $t>t_{0}$ and $h^{1}(t) \leqslant h^{2}(t)$ for $t \in\left[0, t_{0}\right]$. Hence again we have

$$
\frac{d}{d t} h^{1}\left(t_{0}\right)=\frac{d}{d t} h^{2}\left(t_{0}\right), \quad h^{1}\left(t_{0}\right)=h^{2}\left(t_{0}\right), h^{1}(t) \leqslant h^{2}(t) \text { for } t \geqslant 0,
$$

with strict inequality holding for $t>t_{0}$. 
Define

$$
W(t, x)=u^{2}(t, x)-u^{1}(t, x) \quad \text { for }(t, x) \in D:=\left\{(t, x): t>0, x<h^{1}(t)\right\} .
$$

Then we can argue as in the proof of Theorem 3.1 to conclude that

$$
W>0 \text { in } D \text { and } W_{x}\left(t_{0}, h^{1}\left(t_{0}\right)\right)<0 .
$$

It follows that

$$
\frac{d}{d t} h^{1}\left(t_{0}\right)=-\mu_{1} u_{x}^{1}\left(t_{0}, h^{1}\left(t_{0}\right)\right)<-\mu_{1} u_{x}^{2}\left(t_{0}, h^{2}\left(t_{0}\right)\right)=\frac{\mu_{1}}{\mu_{2}} \frac{d}{d t} h^{2}\left(t_{0}\right)<\frac{d}{d t} h^{2}\left(t_{0}\right),
$$

a contradiction to (4.7). This proves that $h^{1}(t)<h^{2}(t)$ for $t>0$.

The proof for the fact that $h^{1}(t)>h^{2}(t)$ for $t<0$ is similar, and we omit the details.

Set

$$
\begin{array}{lll}
\bar{\alpha}:=\sup _{r \geqslant 0} \alpha(r), & \underline{\alpha}:=\inf _{r \geqslant 0} \alpha(r), \\
\bar{\beta}:=\sup _{r \geqslant 0} \beta(r), & \underline{\beta}:=\inf _{r \geqslant 0} \beta(r) .
\end{array}
$$

By Propositions 2.1 and 2.2 in [6], for each $k \in(0,2 \sqrt{\bar{\alpha} d})$, the problem

$$
-d V^{\prime \prime}+k V^{\prime}=V(\bar{\alpha}-\underline{\beta} V) \quad \text { in }(0, \infty), \quad V(0)=0, \quad V(\infty)=\bar{\alpha} / \underline{\beta}
$$

has a unique positive solution $V_{k}(x)$, and for each $\mu>0$, there exists a unique $k_{0}=k_{0}(\mu) \in(0,2 \sqrt{\bar{\alpha} d})$ such that $\mu V_{k_{0}}^{\prime}(0)=k_{0}$. Moreover, $k_{0}(\mu)$ strictly increases to $2 \sqrt{\bar{\alpha} d}$ as $\mu$ increases to $\infty$.

Lemma 4.5. $C(\infty):=\lim _{\mu \rightarrow \infty} C(\mu) \leqslant 2 \sqrt{\bar{\alpha} d}$.

Proof. Denote by $(\bar{u}, \bar{h})$ the unique solution of $(1.1)$ with $(\alpha(r), \beta(r))$ replaced by $(\bar{\alpha}, \beta)$. Since the modified problem is set in a homogeneous environment, we could apply the result of [7] to conclude that

$$
\lim _{t \rightarrow \infty} \frac{\bar{h}(t)}{t}=k_{0}(\mu)<2 \sqrt{\bar{\alpha} d}
$$

for any $\mu>0$.

By the comparison principle in [7], we deduce $h(t) \leqslant \bar{h}(t)$ and $u(t, r) \leqslant \bar{u}(t, r)$ for $t>0$ and $r \in[0, h(t)]$. It follows that $C(\mu) \leqslant k_{0}(\mu)<2 \sqrt{\bar{\alpha} d}$ for all $\mu>0$. Therefore $C(\infty)=\lim _{\mu \rightarrow \infty} C(\mu) \leqslant 2 \sqrt{\bar{\alpha} d}$.

Lemma 4.6. Let $\left(u^{\mu}, h^{\mu}\right)$ be the unique normalized pulsating semi-wave of (1.3). Then

$$
\frac{d}{d t} h^{\mu}(t) \leqslant k_{0}(\mu+1)<2 \sqrt{\bar{\alpha} d} \text { for all } t \in \mathbb{R} .
$$

Proof. Fix an arbitrary $s_{0} \in \mathbb{R}$. We show that there exists $t_{0} \in \mathbb{R}$ such that

$$
h^{\mu}\left(s_{0}\right)=k_{0}(\mu+1) s_{0}+t_{0}, \quad h^{\mu}(t)<k_{0}(\mu+1) t+t_{0} \quad \text { for } t>s_{0} .
$$

Clearly this implies

$$
\frac{d}{d t} h^{\mu}\left(s_{0}\right) \leqslant k_{0}(\mu+1)<2 \sqrt{\bar{\alpha} d} .
$$

Thus it suffices to prove (4.8).

Since

$$
\lim _{t \rightarrow \pm \infty} \frac{h^{\mu}(t)}{t}=C(\mu) \leqslant k_{0}(\mu)<k_{0}(\mu+1),
$$

we have, for any $t_{0} \in \mathbb{R}$, 


$$
\begin{array}{ll}
h^{\mu}(t)<k_{0}(\mu+1) t+t_{0} & \text { for all large positive } t, \\
h^{\mu}(t)>k_{0}(\mu+1) t+t_{0} & \text { for all large negative } t .
\end{array}
$$

Therefore there exists a minimal $s=s\left(t_{0}\right) \in \mathbb{R}$ such that

$$
h^{\mu}(s)=k_{0}(\mu+1) s+t_{0}, \quad h^{\mu}(t) \leqslant k_{0}(\mu+1) t+t_{0} \quad \forall t>s .
$$

We claim that strictly inequality holds, namely

$$
h^{\mu}(t)<k_{0}(\mu+1) t+t_{0} \quad \forall t>s .
$$

Otherwise there exists $s_{1}>s$ such that $h^{\mu}\left(s_{1}\right)=k_{0}(\mu+1) s_{1}+t_{0}$. It then follows that

$$
\frac{d}{d t} h^{\mu}\left(s_{1}\right)=k_{0}(\mu+1) \text {. }
$$

Define $D:=\left\{(t, x): t>s, x<h^{\mu}(t)\right\}$ and

$$
\hat{u}(t, x)=V_{k_{0}(\mu+1)}(\hat{h}(t)-x), \quad \hat{h}(t)=k_{0}(\mu+1) t+t_{0} .
$$

We note that

$$
\hat{u}_{x}(t, \hat{h}(t))=-V_{k_{0}(\mu+1)}^{\prime}(0)<0,
$$

and $u_{x}^{\mu}\left(t, h^{\mu}(t)\right)$ is negative and periodic in $t$. Moreover,

$$
\lim _{\hat{h}(t)-x \rightarrow \infty} \hat{u}(t, x)=\bar{\alpha} / \underline{\beta}, \quad \lim _{h^{\mu}(t)-x \rightarrow \infty}\left[u^{\mu}(t, x)-\phi(x)\right]=0,
$$

where $\phi(x)$ is the unique positive solution of $-d \phi^{\prime \prime}=\phi[a(x)-b(x) \phi]$ in $\mathbb{R}$, and the limits are uniform. Moreover

$$
\underline{\alpha} / \bar{\beta} \leqslant \phi(x) \leqslant \bar{\alpha} / \underline{\beta} .
$$

Therefore there exists $c_{0}>0$ such that

$$
\hat{u}(t, x) \geqslant c_{0} u^{\mu}(t, x) \quad \text { in } D .
$$

Taking the limits along $h^{\mu}(t)-x \rightarrow \infty$ we deduce

$$
c_{0} \underline{\alpha} / \bar{\beta} \leqslant c_{0} \phi(x) \leqslant \bar{\alpha} / \underline{\beta} .
$$

Hence $c_{0} \leqslant \frac{\bar{\alpha} \bar{\beta}}{\alpha \underline{\beta}}$. Define

$$
c^{*}:=\sup \left\{c>0: \hat{u}(t, x) \geqslant c u^{\mu}(t, x) \text { in } D\right\} .
$$

Then

$$
c_{0} \leqslant c^{*} \leqslant \frac{\bar{\alpha} \bar{\beta}}{\underline{\alpha} \underline{\beta}}, \quad \text { and } \quad \hat{u} \geqslant c^{*} u^{\mu} \quad \text { in } D .
$$

We show that $c^{*} \geqslant 1$. Otherwise $c^{*}<1$ and for any positive sequence $\epsilon_{n} \rightarrow 0$, there exists $\left(t_{n}, x_{n}\right) \in D$ such that

$$
\hat{u}\left(t_{n}, x_{n}\right) \leqslant\left(c^{*}+\epsilon_{n}\right) u^{\mu}\left(t_{n}, x_{n}\right), \quad n=1,2, \ldots
$$

Using (4.11) we easily see that $\left\{t_{n}\right\}$ and $\left\{x_{n}\right\}$ are bounded sets in $\mathbb{R}$. We may then argue as in Step 3 of the proof of Theorem 3.1 to reach a contradiction.

Hence $c^{*} \geqslant 1$ and

$$
W(t, x):=\hat{u}(t, x)-u^{\mu}(t, x) \geqslant, \not \equiv 0 \quad \text { in } D, W\left(s_{1}, h^{\mu}\left(s_{1}\right)\right)=0 .
$$

From the equations for $\hat{u}$ and $u^{\mu}$ we see that $W$ satisfies, for some bounded function $c(t, x)$,

$$
W_{t}-d W_{x x}+c(t, x) W \geqslant 0 \text { in } D .
$$

Hence we can apply the strong maximum principle and the Hopf lemma to conclude that

$$
W>0 \text { in } D \text { with } W_{x}\left(s_{1}, h^{\mu}\left(s_{1}\right)\right)<0 .
$$

It follows that

$$
\frac{d}{d t} h^{\mu}\left(s_{1}\right)=-\mu u_{x}^{\mu}\left(s_{1}, h^{\mu}\left(s_{1}\right)\right)<\mu V_{k_{0}(\mu+1)}^{\prime}(0)<(\mu+1) V_{k_{0}(\mu+1)}^{\prime}(0)=k_{0}(\mu+1) .
$$

But this contradicts (4.10). This proves our claim. 
We now examine how $s\left(t_{0}\right)$ varies with $t_{0}$. The property of $s\left(t_{0}\right)$ proved in the above claim implies that it varies continuously with $t_{0} \in \mathbb{R}$. By (4.9), we see that

$$
\lim _{t_{0} \rightarrow+\infty} s\left(t_{0}\right)=-\infty, \quad \lim _{t_{0} \rightarrow-\infty} s\left(t_{0}\right)=+\infty .
$$

Hence for each $s_{0} \in \mathbb{R}$, there exists $t_{0}$ such that $s\left(t_{0}\right)=s_{0}$. The conclusions in (4.8) now follow from the claim.

Lemma 4.7. $C(\infty)=c_{*}$.

Proof. Let $\left\{\mu_{n}\right\}$ be an increasing sequence converging to $\infty$ as $n \rightarrow \infty$. To simplify notations we write $\left(u^{n}, h^{n}\right)$ for the unique normalized pulsating semi-wave $\left(u^{\mu_{n}}, h^{\mu_{n}}\right)$ of (1.3) with $\mu=\mu_{n}$, and denote by $\left(U^{n}(\tau, \xi), p^{n}(\tau)\right)$ the corresponding pair of $\left(u^{n}(t, x), h^{n}(t)\right)$.

Making use of Lemma 4.6 and $p^{n}\left(h^{n}(t)\right)=\frac{d}{d t} h^{n}(t)$, we obtain $p^{n}(\tau)<2 \sqrt{\bar{\alpha} d}$ for all $\tau \in R$ and $n \geqslant 1$. Moreover, since $\left\{\mu_{n}\right\}$ is increasing, by Lemma 4.4 and using suitable shifts, it is easily seen that if $h^{n}(t)=h^{n+1}\left(t^{\prime}\right)$, then $\frac{d}{d t} h^{n}(t) \leqslant \frac{d}{d t} h^{n+1}\left(t^{\prime}\right)$. It follows that $p^{n}(\tau) \leqslant p^{n+1}(\tau)$ for all $\tau \in \mathbb{R}$. Therefore we may assume that $p^{n} \rightarrow p^{*}$ with

$$
p^{1}(\tau) \leqslant p^{*}(\tau) \leqslant 2 \sqrt{\bar{\alpha} d}, \quad p^{*}(\tau)=p^{*}(\tau+L) \quad \forall \tau \in \mathbb{R} .
$$

As in the proof of Theorem 3.4, by a simple comparison consideration we see that $\left\{U^{n}\right\}$ is a bounded set in $L^{\infty}(\mathbb{R} \times$ $[0, \infty)$ ). Therefore we may apply the $L^{p}$ theory for parabolic equations to the equation of $U^{n}$, much as in the proof of Theorem 3.4, to conclude that, by passing to a subsequence, $U^{n} \rightarrow U^{*}$ in $C_{l o c}^{\frac{v+1}{2}, v+1}([0, L] \times[0, \infty))$, and $\left(U^{*}, p^{*}\right)$ satisfies

$$
\begin{aligned}
& p^{*}(\tau)\left(U_{\tau}^{*}+U_{\xi}^{*}\right)-d U_{\xi \xi}^{*}=U^{*}\left[a(\tau-\xi)-b(\tau-\xi) U^{*}\right), \quad(\tau, \xi) \in \mathbb{R} \times[0, \infty), \\
& U^{*}(\tau, 0)=0, \quad \tau \in \mathbb{R} .
\end{aligned}
$$

From the identity $p^{n}(\tau)=\mu_{n} U_{\xi}^{n}(\tau, 0)$, we deduce

$$
U_{\xi}^{*}(\tau, 0) \equiv 0 .
$$

Since $U^{*} \geqslant 0$, the Hopf lemma infers that $U^{*} \equiv 0$.

We now examine the sequence $\left\{\left(u^{n}, h^{n}\right)\right\}$. Let us note that for any $t_{0} \in \mathbb{R},\left(u^{n}\left(t+t_{0}, x\right), h^{n}\left(t+t_{0}\right)\right)$ is also a pulsating semi-wave to (1.3) with $\mu=\mu_{n}$. For fixed $n \geqslant 1$, since $u^{n}(0,0)=0, \lim _{t \rightarrow \infty} u^{n}(t, 0)=\phi(0) \geqslant \underline{\alpha} / \bar{\beta}$, and $u^{n}(t, x)$ is strictly increasing in $t$, for fixed $\zeta \in(0, \underline{\alpha} / \bar{\beta})$, there exists a unique $t_{n}>0$ such that $u^{n}\left(t_{n}, 0\right)=\zeta$. We now replace $\left(u^{n}, h^{n}\right)$ by its shift $\left(u^{n}\left(t+t_{n}, x\right), h^{n}\left(t+t_{n}\right)\right)$, and still denote it by $\left(u^{n}, h^{n}\right)$. Then clearly

$$
u^{n}(0,0)=\zeta>0, \quad h^{n}(0)>0 \quad \forall n \geqslant 1 .
$$

We claim that $t_{n} \rightarrow \infty$ as $n \rightarrow \infty$. Otherwise by passing to a subsequence we may assume that $t_{n} \rightarrow t^{*}$. Then from the boundedness of $\left\{\frac{d}{d t} h^{n}\right\}$ we conclude that, subject to a subsequence, $h^{n} \rightarrow h^{*}$ in $C_{l o c}^{\mu}(\mathbb{R})$. Since $\left\|u_{n}\right\|_{\infty}=\left\|U^{n}\right\|_{\infty}$ has a bound independent of $n$, we may apply $L^{p}$ theory to the equation of $u^{n}$ to conclude that, by passing to a subsequence, $u^{n} \rightarrow u^{*}$ in $C_{l o c}^{\frac{1+v}{2}, 1+v}(D)$, with $D=\left\{(t, x): t \in \mathbb{R}, x<h^{*}(t)\right\}$. Moreover, we have $u^{*}(0,0)=\zeta>0$. On the other hand, by the discussion above for the corresponding pairs $\left(U^{n}, p^{n}\right)$, we must have $u^{*} \equiv 0$. This contradiction shows that $t_{n} \rightarrow \infty$, as claimed.

Clearly the above proved claim implies that $h^{n}(t) \rightarrow \infty$ as $n \rightarrow \infty$, uniformly for $t \in[-M, \infty)$ for every $M>0$. We may now once more apply the $L^{p}$ theory to the equation of $u^{n}$ to conclude that, by passing to a subsequence, $u^{n} \rightarrow u^{*}$ in $C_{l o c}^{\frac{1+v}{2}, 1+v}\left(R^{2}\right)$, with $u^{*}(0,0)=\zeta>0$. Moreover, $u^{*}$ satisfies

$$
u_{t}^{*}-d u_{x x}^{*}=u^{*}\left[a(x)-b(x) u^{*}\right] \text { for }(t, x) \in \mathbb{R}^{2} .
$$

Recall that $\frac{d}{d t} h^{n}(t)$ is $T_{n}$-periodic, with $C\left(\mu_{n}\right)=L / T_{n}$, and $u^{n}\left(t+T_{n}, x\right)=u^{n}(t, x-L)$. Since $C\left(\mu_{n}\right) \rightarrow C(\infty)$, we obtain $T_{n} \rightarrow T^{*}:=L / C(\infty)$. It follows that

$$
u^{*}\left(t+T^{*}, x\right)=u^{*}(t, x-L) \quad \text { for all }(t, x) \in \mathbb{R}^{2} .
$$

Due to $u_{t}^{n}(t, x)>0$, we obtain $u_{t}^{*}(t, x) \geqslant 0$. By the strong maximum principle, we easily see that either $u_{t}^{*}>0$ or $u_{t}^{*} \equiv 0$. If $u_{t}^{*} \equiv 0$, then necessarily $u^{*}(t, x)=\phi(x)$. But we have $u^{*}(0,0)=\zeta<\min \phi(x)$. Hence we necessarily have 
$u_{t}^{*}(t, x)>0$. From these properties of $u^{*}$ we can now conclude that $u^{*}$ is a pulsating front of (1.4). By (4.12), the speed of $u^{*}$ is $C(\infty)$. Therefore we must have $C(\infty) \geqslant c_{*}$.

We show next that $C(\infty) \leqslant c_{*}$. For this purpose, we consider the auxiliary one-dimensional free boundary problem

$$
\begin{cases}u_{t}-d u_{x x}=u[a(x)-b(x) u], & t>0, x \in(g(t), h(t)), \\ u(t, g(t))=0, \quad g^{\prime}(t)=-\mu u_{x}(t, g(t)), & t>0, \\ u(t, h(t))=0, \quad h^{\prime}(t)=-\mu u_{x}(t, h(t)), & t>0, \\ -g(0)=h(0)=h_{0}, \quad u(0, x)=u_{0}(x), & x \in\left[-h_{0}, h_{0}\right] .\end{cases}
$$

Comparing (4.13) with the same problem where $(a(x), b(x))$ is replaced by the constant pair $(\min a(x), \max b(x))$, one easily sees from [10] that for all large $\mu>0$, spreading occurs for (4.13). Moreover, a similar (but easier) analysis to that of Theorem 4.12 shows that

$$
\lim _{t \rightarrow \infty} \frac{-g(t)}{t}=\lim _{t \rightarrow \infty} \frac{h(t)}{t}=C(\mu) .
$$

Let $\hat{u}(t, x)$ be the unique solution of the corresponding Cauchy problem to (4.13). Then by the comparison principle we have $u(t, x)<\hat{u}(t, x)$, which implies that $C(\mu)$ is no bigger than the spreading speed determined by this Cauchy problem. By [2] the spreading speed determined by this Cauchy problem is the minimal speed of the pulsating fronts of (1.4), namely $c_{*}$. Therefore we have $C(\mu) \leqslant c_{*}$. It follows that $C(\infty) \leqslant c_{*}$.

\section{References}

[1] H. Berestycki, F. Hamel, Front propagation in periodic excitable media, Commun. Pure Appl. Math. 55 (2002) 949-1032.

[2] H. Berestycki, F. Hamel, G. Nadin, Asymptotic spreading in heterogeneous diffusive excitable media, J. Funct. Anal. 255 (9) (2008) 2146-2189.

[3] H. Berestycki, F. Hamel, N. Nadirashvili, The speed of propagation for KPP type problems. I. Periodic framework, J. Eur. Math. Soc. 7 (2005) $173-213$.

[4] H. Berestycki, F. Hamel, L. Roques, Analysis of the periodically fragmented environment model. I. Species persistence, J. Math. Biol. 51 (2005) 75-113.

[5] H. Berestycki, F. Hamel, L. Roques, Analysis of the periodically fragmented environment model. II. Biological invasions and pulsating travelling fronts, J. Math. Pures Appl. 84 (2005) 1101-1146.

[6] G. Bunting, Y. Du, K. Krakowski, Spreading speed revisited: analysis of a free boundary model, Netw. Heterog. Media 7 (2012) $583-603$.

[7] Y. Du, Z.M. Guo, Spreading-vanishing dichotomy in the diffusive logistic model with a free boundary II, J. Diff. Eqns. 250 (2011) $4336-4366$.

[8] Y. Du, Z.M. Guo, The Stefan problem for the Fisher-KPP equation, J. Diff. Eqns. 253 (2012) 996-1035.

[9] Y. Du, Z.M. Guo, R. Peng, A diffusive logistic model with a free boundary in time-periodic environment, J. Funct. Anal. 265 (2013) 2089-2142.

[10] Y. Du, Z.G. Lin, Spreading-vanishing dichotomy in the diffusive logistic model with a free boundary, SIAM J. Math. Anal. 42 (2010) 377-405; SIAM J. Math. Anal. 45 (2013) 1995-1996 (Erratum).

[11] Y. Du, L.S. Liu, Remarks on the uniqueness problem for the logistic equation on the entire space, Bull. Aust. Math. Soc. 73 (2006) $129-137$.

[12] A. Ducrot, T. Giletti, H. Matano, Existence and convergence to a propagating terrace in one-dimensional reaction-diffusion equations, Trans. Am. Math. Soc. (2013), in press.

[13] J. Gärtner, M.I. Freidlin, On the propagation of concentration waves in periodic and random media, Sov. Math. Dokl. 20 (1979) $1282-1286$.

[14] F. Hamel, L. Roques, Uniqueness and stability properties of monostable pulsating fronts, J. Eur. Math. Soc. 13 (2011) $345-390$.

[15] O.A. Ladyzenskaja, V.A. Solonnikov, N.N. Ural'ceva, Linear and Quasilinear Equations of Parabolic Type, Amer. Math. Soc., Providence, RI, 1968

[16] X. Liang, X.-Q. Zhao, Spreading speeds and traveling waves for abstract monostable evolution systems, J. Funct. Anal. 259 (4) (2010) 857-903.

[17] G.M. Lieberman, Second Order Parabolic Differential Equations, World Scientific, Singapore, 1996.

[18] H. Matano, Traveling waves in spatially random media, in: Mathematical Economics, in: RIMS Kokyuroku, vol. 1337, 2003 , pp. 1-9.

[19] G. Nadin, The principal eigenvalue of a space-time periodic parabolic operator, Ann. Mat. Pura Appl. 188 (2009) $269-295$.

[20] G. Nadin, Existence and uniqueness of the solution of a space-time periodic reaction-diffusion equation, J. Diff. Eqns. 249 (2010) $1288-1304$.

[21] Wenxian Shen, Traveling waves in time dependent bistable equations, Diff. Integral Eqns. 19 (2006) 241-278.

[22] H.F. Weinberger, On spreading speeds and traveling waves for growth and migration models in a periodic habitat, J. Math. Biol. 45 (6) (2002) $511-548$.

[23] Maolin Zhou, The asymptotic behavior of the Fisher-KPP equation with free boundary, preprint. 